\title{
Phase Locking of Auditory Nerve Fibers: The Role of Lowpass Filtering by Hair Cells
}

\author{
Adam J. Peterson ${ }^{1}$ and ${ }^{\circledR}$ Peter Heil ${ }^{1,2}$ \\ ${ }^{1}$ Leibniz Institute for Neurobiology, Magdeburg, 39118, Germany, and ${ }^{2}$ Center for Behavioral Brain Sciences, Magdeburg, 39106, Germany
}

Phase locking of auditory-nerve-fiber (ANF) responses to the temporal fine structure of acoustic stimuli, a hallmark of the auditory system's temporal precision, is important for many aspects of hearing. Previous work has shown that phase-locked period histograms are often well described by exponential transfer functions relating instantaneous stimulus pressure to instantaneous spike rate, with no observed clipping of the histograms. The operating points and slopes of these functions change with stimulus level. The mechanism underlying this apparent gain control is unclear but is distinct from mechanical compression, is independent of refractoriness and spike-rate adaptation, and is apparently instantaneous. Here we show that these findings can be accounted for by a model consisting of a static Boltzmann transducer function yielding a clipped output, followed by a lowpass filter and a static exponential transfer function. Using responses to tones of ANFs from cats of both sexes, we show that, for a given ANF, the period histograms obtained at all stimulus levels for a given stimulus frequency can be described using one set of levelindependent model parameters. The model also accounts for changes in the maximum and minimum instantaneous spike rates with changes in stimulus level. Notably, the estimated cutoff frequency is lower for low- than for high-spontaneous-rate ANFs, implying a synapse-specific contribution to lowpass filtering. These findings advance our understanding of ANF phase locking by highlighting the role of peripheral filtering mechanisms in shaping responses of individual ANFs.

Key words: auditory nerve; Boltzmann function; lowpass filter; modeling; phase locking; ribbon synapse

\section{Significance Statement}

Phase locking of auditory-nerve-fiber responses to the temporal fine structure of acoustic stimuli is important for many aspects of hearing. Period histograms typically retain an approximately sinusoidal shape across stimulus levels, with the peripheral auditory system operating as though its overall transfer function is an exponential function whose slope decreases with increasing stimulus level. This apparent gain control can be accounted for by a static saturating transducer function followed by a lowpass filter. In addition to attenuating the AC component, the filter approximately recovers the sinusoidal waveform of the stimulus. The estimated cutoff frequency varies with spontaneous rate, revealing a synaptic contribution to lowpass filtering. These findings highlight the significant impact of peripheral filtering mechanisms on phase locking.

\section{Introduction}

Primary auditory afferents (auditory-nerve fibers [ANFs]) show phase locking in response to low-frequency tones, low-frequency components of broadband sounds, and low-frequency fluctuations of envelopes (for review, see Joris et al., 2004; Heil and Peterson,

Received Sep. 20, 2019; revised Feb. 13, 2020; accepted Apr. 22, 2020.

Author contributions: A.J.P. analyzed data; A.J.P. and P.H. wrote the paper; P.H. designed research; P.H. performed research.

This work was supported by Deutsche Forschungsgemeinschaft Priority Program 1608 "Ultrafast and temporally precise information processing: normal and dysfunctional hearing" (He1721/11-2) to P.H. P.H. was also supported by Deutsche Forschungsgemeinschaft Grants He1721/5-1 and He1721/5-2. Data collection was done in the laboratory of Dexter R.F. Irvine (Monash University, Clayton, Victoria, Australia). We thank Dexter R.F. Irvine and Mel Brown for help with data collection; and Laurel Carney for suggesting the idea pursued in this study.

The authors declare no competing financial interests.

Correspondence should be addressed to Peter Heil at peter.heil@lin-magdeburg.de.

https://doi.org/10.1523/JNEUROSCI.2269-19.2020

Copyright $\odot 2020$ the authors
2015, 2017). Phase locking to the fine structure of acoustic stimuli is thought to be important for sound localization (Grothe et al., 2010), sound detection (Huet et al., 2018), and pitch perception and speech understanding (Lorenzi et al., 2006; Moore, 2008; Verschooten et al., 2019) in quiet and in background noise.

Period histograms from phase-locked ANF responses to lowfrequency tones show no sign of peak clipping at high stimulus levels, even beyond that at which the mean spike rate has saturated. Peak clipping would prevent the instantaneous spike rate from coding the full range of instantaneous pressures. The lack of peak clipping is perhaps surprising because apical cochlear mechanical responses show little, if any, compression (Robles and Ruggero, 2001), and hair-cell transduction is thought to obey a static saturating sigmoidal nonlinearity (Meddis, 2006; Zilany et al., 2009; Altoè et al., 2018; Horst et al., 2018). Many period histograms are well described by a sinusoidal stimulus passed through an exponential transfer function (Colburn, 1973; Johnson, 1974; Horst et al., 2018; Peterson and Heil, 2019). 
Others require adding distortion components to the stimulus (Johnson, 1974; Peterson and Heil, 2019). The operating point of the exponential function (the instantaneous rate at an instantaneous pressure of $0 \mathrm{~Pa}$ ) often increases with increasing stimulus level (Peterson and Heil, 2019). In contrast, the slope of the exponential function is approximately constant across low stimulus levels (Horst et al., 2018) and then decreases with increasing level (Peterson and Heil, 2019). This change in the slope represents an apparent gain control that prevents peak clipping at high stimulus levels.

The mechanism underlying the change in slope is unknown. Peterson and Heil (2019) showed that neither ANF refractoriness nor mechanisms responsible for spike-rate adaptation on time scales longer than the stimulus period are involved. They also demonstrated that the mechanism must be fast because the slope is set already during the first cycles of the response. Two potential mechanisms for this change in slope have been suggested. One is a change of the microscopic stiffness of the hypothetical gating spring in the transduction machinery in the inner-haircell stereocilia, such that a larger displacement of the stereocilia is required at high stimulus levels than at low stimulus levels to yield a given open probability of the mechanoelectrical transducer (MET) channels. Such a mechanism, corresponding to a transducer function whose slope decreases with increasing level, has been proposed for basal outer hair cells (Patuzzi and Moleirinho, 1998; Meenderink and van der Heijden, 2011). The other potential mechanism, proposed by Laurel Carney (personal communication), is a static saturating transducer function that produces clipped MET currents, followed by a lowpass filter that converts the currents into approximately sinusoidal signals.

Russell et al. (1986a) recorded membrane potentials of isolated inner hair cells during sinusoidal displacements of their stereocilia bundles. Their data (e.g., their Fig. 5) reveal that increasing the amplitude of the sinusoidal displacement increases the instantaneous displacement needed to produce a given receptor potential, which is consistent with either mechanism. Some published recordings of MET currents from inner hair cells in the hemicochlea (Jia et al., 2007) or hair cells in vitro (Fettiplace and Kim, 2014) display responses consistent with a static Boltzmann transducer function.

Here, we investigate the feasibility of the second proposed mechanism by fitting period histograms with a model consisting of a static Boltzmann transducer function followed by a lowpass filter and a static exponential transfer function. For a given ANF and stimulus frequency, one set of model parameters is fitted to the period histograms obtained for all stimulus levels, such that all model components are level independent. We find that such a model accounts well for the data.

\section{Materials and Methods}

The data for this study consist of responses of 77 ANFs in 5 barbiturateanesthetized adult cats ( 3 females, 2 males; weighing 3-3.5 kg) to lowfrequency tones (typically $100 \mathrm{~ms}$, including 4.2 -ms-long cosine-squared rise and fall times). Tones of a selected frequency were presented at a range of sound pressure levels (SPLs) increasing from low to high (typically in $4 \mathrm{~dB}$ steps from below rate threshold to $80-100 \mathrm{~dB}$ SPL), referred to here as a "level series." A given stimulus level was repeated up to 200 times (typically 50 times) at a fixed rate (typically $4 \mathrm{~Hz}$ ) before advancing to the next level in the series. The spontaneous activity of the ANF was recorded last. If the recording conditions were still stable, another stimulus (typically differing in frequency) was selected and the protocol was repeated. Information about surgical and recording procedures is provided in Peterson and Heil (2019) and will not be repeated here. All procedures were approved by the Monash University Department of Psychology Animal Ethics Committee. The data used here are identical to those in Peterson and Heil (2019) and consist of period histograms from which the effects of ANF refractoriness were removed to yield an estimate of the instantaneous rate of transmitter release events at the inner-hair-cell ribbon synapse.

\section{Data analysis}

Data analysis was performed using MATLAB 2019 (The MathWorks).

Estimating the instantaneous rate of release events. We adapted the method of Johnson and Swami (1983) to estimate and remove the effects of refractoriness from period histograms of spikes to obtain the "free firing rate" (Berry and Meister, 1998; Avissar et al., 2013), the spike rate expected if the ANF were free from refractory effects. Here, we refer to this rate as the "event rate" because it also corresponds to the rate of transmitter release events from the ribbon synapse in the inner hair cell that drives the ANF, assuming every release event triggers a spike when the ANF is not refractory. The refractory period following each spike was modeled as a fixed dead time having duration $t_{\mathrm{D}} \geq 0$ followed by a random dead time whose duration was drawn from an exponential distribution with mean $t_{\mathrm{R}}>0$. The probability $p$ that the ANF is excitable (i.e., is not refractory) at time $t^{\prime}$ since the previous spike is as follows:

$$
\begin{gathered}
p\left(t^{\prime}\right)=1-\mathrm{e}^{-\left(t^{\prime}-t_{D}\right) / t_{R}} \text { for } t^{\prime} \geq t_{\mathrm{D}} \\
p\left(t^{\prime}\right)=0 \text { for } t^{\prime}<t_{\mathrm{D}} .
\end{gathered}
$$

For a given spike train (i.e., one continuous recording of spike times, including all stimulus repetitions and silent interstimulus intervals), one continuous excitability function was computed with the excitability equaling 1 before the first spike and following the time course described by Equation 1 after each spike. Both $t_{\mathrm{D}}$ and $t_{\mathrm{R}}$ were set to $0.6 \mathrm{~ms}$, consistent with previous estimates (Heil et al., 2007; Peterson and Heil, 2018; Peterson et al., 2018). The time course of the mean excitability during a cycle was computed analogously to the period histogram representing the instantaneous spike rate. Both the period histogram and the mean excitability used only the portions of the response corresponding to complete cycles of the stimulus, starting from $10 \mathrm{~ms}$ after stimulus onset to exclude onset effects. The period histogram representing the estimated instantaneous event rate was then computed by dividing the period histogram of the spikes by the mean excitability function. Because the mean excitability function can take any value between 0 and 1 , the event counts underlying the estimated event rates are typically noninteger.

\section{Model fitting}

We used maximum likelihood estimation to fit a model of phase locking (see Results) to the instantaneous event rate. For simplicity, we assume that, during sound stimulation, the event generating process is an inhomogeneous Poisson process. Because the instantaneous event rate typically corresponds to noninteger numbers of events in each bin of a period histogram, we computed likelihoods using the continuous approximation of the discrete Poisson distribution, $f(n \mid \lambda)=\lambda^{n} \mathrm{e}^{-\lambda} / \Gamma(n+1)$, where $n$ is the number of events in a particular bin, $\lambda$ is the expected number of events in that bin, $\Gamma$ is the gamma function, and $f$ is the probability of observing $n$ given $\lambda$. Before computing likelihoods, the period histograms from both the model and the data were shifted to align the phases of their mean vectors at $\pi$ to avoid having to include a phase parameter for each histogram.

The goal of the present study was to account for the level-dependent changes in the period histograms of a given ANF and stimulus frequency using a model with only level-independent parameters. We therefore sought the set of parameter values yielding the maximum joint probability (i.e., the maximum likelihood) across all bins of all period histograms from the level series that met our inclusion criteria. Histograms were included only if based on $\geq 125$ spikes and if the vector strength was significant (Rayleigh test of uniformity, $p<0.01$ ). For each period histogram, the expected event count per bin, $\lambda$, was computed from the model's rate equation (Eq. 9). For $z$ period histograms, each containing 
$m$ bins, event counts are denoted $n_{1,1}, n_{1,2}, \ldots, n_{z, m}$. The negative log likelihood, $-\ln \mathcal{L}$, of a set of parameter values $\theta_{\lambda}$ that defines the expected event counts $\lambda_{1,1}, \lambda_{1,2}, \ldots, \lambda_{z, m}$ is given by the following:

$$
\begin{aligned}
-\ln \mathcal{L}\left(\theta_{\lambda} \mid n_{1,1}, n_{1,2}, \ldots, n_{z, m}\right) & =-\ln \prod_{j=1}^{z} \prod_{k=1}^{m} f\left(n_{j, k} \mid \lambda_{j, k}\right) \\
& =-\sum_{j=1}^{z} \sum_{k=1}^{m} \ln f\left(n_{j, k} \mid \lambda_{j, k}\right) .
\end{aligned}
$$

The set of model parameters yielding the minimum negative log likelihood was estimated using the MATLAB function fmincon. There are three model parameters for which a change in value requires recomputing the output of the lowpass filter (see Eqs. 6, 7): $M_{0}$, the normalized MET current at rest (equal to the resting open probability of the MET channels); $b$, the slope factor of the transducer function; and $f_{c}$, the cutoff frequency of the lowpass filter. Because filtering is computationally demanding, we used a grid-search approach to minimize the number of times the filter output had to be computed during fitting. First, $M_{0}$ was fixed to 1 of 19 linearly spaced values over the interval from 0.05 to 0.95 . Next, a grid was defined with 31 values of $b$ logarithmically spaced over the interval from 1 to $100,000 \mathrm{~Pa}^{-1}$ and 61 values of $f_{\mathrm{c}}$ logarithmically spaced values over the interval from $f_{1} / 10$ to $f_{1} \cdot 10 \mathrm{~Hz}$, where $f_{1}$ is the stimulus frequency. For the selected $M_{0}$ and each of the $31 \times 61$ pairs of $b$ and $f_{\mathcal{c}}$, the slope factor of the exponential function, parameter $D$ (see Eq. 9), was then fitted. Because there was sometimes one minimum in the region $f_{\mathrm{c}}<f_{1}$ and another, distinct, minimum in the region $f_{\mathrm{c}}>f_{1}$, a "best pair" (i.e., the pair of $b$ and $f_{c}$ yielding the smallest negative log likelihood) was identified within each region. Further fits were performed while iteratively zooming in to the region of the grid near each best pair until the values of $b$ and $f_{c}$ each changed by $<1 \%$ between adjacent points. The combination of $b, f_{c}$, and $D$ yielding the smallest negative log likelihood was chosen as the optimum set of parameter values for the selected value of $M_{0}$. The entire process was then repeated for each remaining value of $M_{0}$. The combination of $M_{0}, b, f_{c}$, and $D$ yielding the smallest overall negative log likelihood was taken as the global minimum.

To verify the reliability of the fitting procedure, period histograms were simulated using known model parameters and then fitted by the model using the grid-based procedure. The simulated histograms were produced by taking the best-fitting model functions, computing the expected event count per bin, and drawing random counts from Poisson distributions having these expected values. Such simulations were performed for several real ANF level series, including examples having different spontaneous rates (SRs) and different stimulus frequencies. In most cases examined, the parameter estimates closely agreed with the known values, implying that the fitting procedure is reliable. In other cases, a broad range of $M_{0}$ values yielded negative log likelihoods near the global minimum, such that nearly identical model functions were obtained for different parameter combinations. In these cases, the parameter estimates obtained from the global minimum sometimes did not match the known values, despite the model functions closely matching the simulated histograms. A similar finding was made when fitting the real data and is addressed below (see Population results; see Figs. $6 B, 7 D-F$ ).

Code accessibility

The code for the model and for the grid-based fitting procedure is accessible online (Peterson, 2020).

\section{Results}

\section{Model}

Peterson and Heil (2019) found that period histograms of instantaneous event rates, $R(t)$ (estimated by removing the effects of refractoriness from the instantaneous spike rates; see Materials and Methods), could be well described by a mechanical drive, $P(t)$, passed through an exponential transfer function. The waveform of the mechanical drive is given by the following:

$$
\begin{gathered}
P(t)=P_{1} \cdot \sin \left(2 \pi f_{1} t+\varphi_{1}\right)+P_{2} \cdot \sin \left(2 \pi f_{2} t+\varphi_{2}\right) \\
+P_{3} \cdot \sin \left(2 \pi f_{3} t+\varphi_{3}\right)
\end{gathered}
$$

where $P_{1}, f_{1}$, and $\varphi_{1}$ specify the amplitude (in $\mathrm{Pa}$ ), frequency (in $\mathrm{Hz}$ ), and phase (in $\mathrm{rad}$ ), respectively, of the stimulus, and $P_{2}, P_{3}, f_{2}=2 \cdot f_{1}, f_{3}=3 \cdot f_{1}, \varphi_{2}$, and $\varphi_{3}$ specify the amplitudes, frequencies, and phases, respectively, of two harmonic distortion components. The distortions were included to account for the temporal asymmetry of period histograms, including peak splitting. The exponential transfer function is given by the following:

$$
R(t)=A \cdot \mathrm{e}^{B \cdot P(t)}
$$

where $A$ is the scale factor that specifies the rate (in events/s) at $P(t)=0 \mathrm{~Pa}$ (i.e., the operating point) and $B$ is the slope factor (in $\left.\mathrm{Pa}^{-1}\right)$. Peterson and Heil (2019) observed that the slope factor $B$ and operating point $A$ vary with stimulus level.

Here, we attempt to account for these findings by means of an extended model that has no level-dependent parameters. This extended model consists of a static Boltzmann transducer function followed by a lowpass filter and a static exponential transfer function. To keep the number of model parameters small, we omitted the distortion components used by Peterson and Heil (2019).

Figure 1 shows the input, transfer function, and output of each stage of the extended model for one stimulus frequency and several stimulus levels. The instantaneous stimulus pressure is given by the following:

$$
P(t)=P_{1} \cdot \sin \left(2 \pi f_{1} t+\varphi_{1}\right)
$$

Figure $1 A$ shows one cycle of the instantaneous pressure, with $\varphi_{1}=0$, for 13 stimuli ranging from 30 to $78 \mathrm{~dB}$ SPL in steps of $4 \mathrm{~dB}$. We chose a first-order Boltzmann function as the static saturating transducer function that relates the instantaneous stimulus pressure to the instantaneous open probability of the MET channels. The current through the MET channels is assumed to be proportional to the open probability. A first-order Boltzmann function describes the open probability of a two-state channel that transitions between a closed and an open state. It accounts well for transfer functions relating basilar membrane displacement (which in the apical cochlea is approximately proportional to the instantaneous stimulus pressure) (Robles and Ruggero, 2001) to MET currents in inner hair cells (e.g., Jia et al., 2007, their Fig. 1). Although Fettiplace and Kim (2014) note that several ex vivo datasets are better described by a second-order (three-state) Boltzmann function, they express concern that the need for such a function (and the more gradual curvature at its upper end) might be an artifact of the mechanical stimulation technique. They suggest that such an artifact would particularly affect inner hair cells due to the morphology of their hair bundles. The instantaneous normalized MET current (defined as the unitless ratio of the current to the maximum possible current and equal to the instantaneous open probability) is therefore given by the following:

$$
M(t)=\frac{1}{1+\left(1 / M_{0}-1\right) \cdot \mathrm{e}^{-b \cdot P(t)}}
$$

where $M_{0}$ is the normalized current at rest (equal to the resting open probability) and $b(>0)$ is the slope factor (in $\mathrm{Pa}^{-1}$ ). 


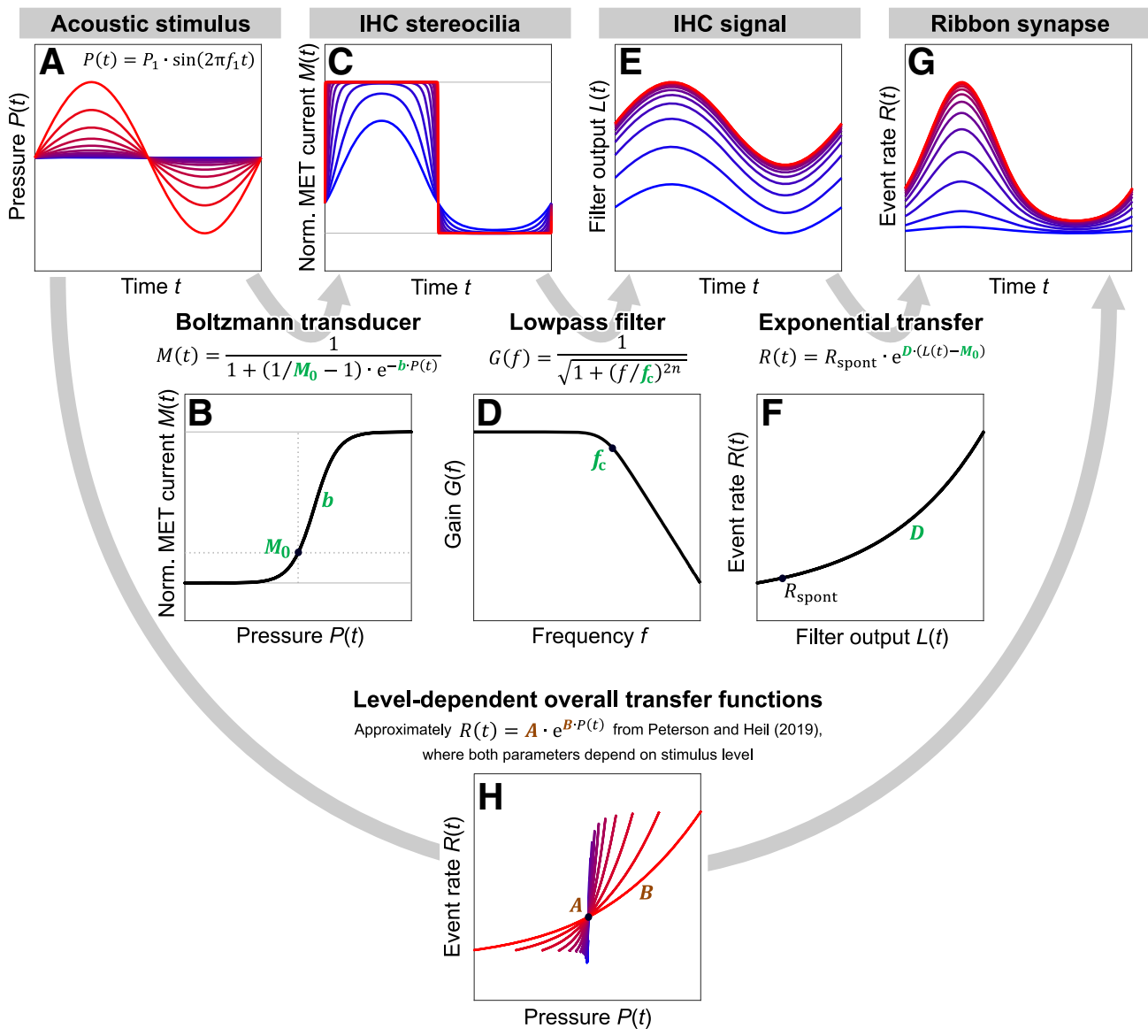

Figure 1. Input, transfer function, and output of each stage of the model for one stimulus frequency and several stimulus levels. $A$, One cycle of the instantaneous pressure for each stimulus. The 13 stimuli range from 30 to $78 \mathrm{~dB}$ SPL in steps of $4 \mathrm{~dB}$. B. First-order Boltzmann function relating the instantaneous stimulus pressure to the normalized MET current (or, equivalently, to the open probability of the MET channels), with $M_{0}=0.20$ and $b=2743 \mathrm{~Pa}^{-1}$. C, Instantaneous normalized MET currents for the stimuli in $\boldsymbol{A}$. D, Third-order Butterworth lowpass filter with $f_{d}$, $f_{1}=0.54$. $E$, Instantaneous filter outputs for the stimuli in $\boldsymbol{A}$. These outputs are approximately sinusoidal for all stimulus levels, but saturate at the higher levels corresponding to the saturation of the MET current. The phase of each output was shifted to align the maximum output to the maximum stimulus pressure. $\boldsymbol{F}$, Exponential transfer function relating the instantaneous filter output to the event rate. $\boldsymbol{G}$, Instantaneous event rates resulting from the stimuli in $\boldsymbol{A}$. $\boldsymbol{H}$, Overall transfer functions relating the instantaneous stimulus pressure to the event rate. They are approximately exponential and have level-dependent slopes and operating points, despite the model having no level-dependent parameters.

Because $b$ can also be conceived of as a scale factor for the input to the Boltzmann function, and because $b$ is constant across levels, the model lacks any level-dependent amplification of the stimulus, such as might occur from a cochlear amplifier. Figure $1 B$ shows a first-order Boltzmann function with $M_{0}=0.20$ and $b=2743 \mathrm{~Pa}^{-1}$. Figure $1 C$ shows the resulting instantaneous normalized MET currents for the stimuli in Figure $1 A$. As the stimulus level increases, the maximum current initially increases before saturating, the minimum current initially decreases before saturating, and the peaks and troughs become increasingly clipped. These behaviors are consistent with the saturation and clipping observed in experimental data (e.g., for inner hair cells, see Jia et al., 2007, their Fig. 1C; for an outer hair cell, see Fettiplace and Kim, 2014, their Fig. 4D,E).

There are likely several lowpass filtering stages between the stimulus and transmitter release. Kidd and Weiss (1990) argue for at least three, because, in addition to the membrane capacitance, the entry and accumulation of calcium required to trigger release at the synapse might also act as lowpass filters. To keep the number of model parameters small, we use a single thirdorder Butterworth lowpass filter, located after the normalized MET currents. The gain $G$ of the frequency response of a Butterworth lowpass filter is given by the following:

$$
G(f)=\frac{1}{\sqrt{1+\left(\frac{f}{f_{c}}\right)^{2 n}}}
$$

where $f$ is the frequency, $f_{\mathrm{c}}$ is the cutoff frequency, and $n$ is the filter order. Figure $1 D$ shows the transfer function of such a filter with $n=3$. Filtering was applied in the forward direction only using the MATLAB function filter. Several cycles of the MET current were computed and filtered, and one cycle from the steady-state portion of the filter output was retained. Figure $1 E$ shows the instantaneous outputs, $L(t)$, resulting from a cutoff frequency of $0.54 \times$ the stimulus frequency. For display purposes, the phase shifts introduced by the model's filter were compensated by shifting each output to align the phase of the mean vector to the maximum stimulus pressure. In this example, the outputs are approximately sinusoidal for all stimulus levels but saturate at the higher levels corresponding to the saturation of the MET current. For a given stimulus level, the mean value of the normalized MET current (Fig. 1C) and the mean value of the filter output (Fig. $1 E$ ) are identical. For an instantaneous pressure of $0 \mathrm{~Pa}$, the instantaneous value of the filter output changes (here, increases) with stimulus level, whereas the normalized MET current at rest is constant (Fig. 1C). The filter output does not correspond perfectly to the membrane potential because our 
single filter combines the filtering performed by the membrane capacitance and by the synapse. The filtering performed by the membrane capacitance likely dominates, such that the filter output should nonetheless be similar to the membrane potential. Indeed, the approximately sinusoidal shape and the saturation of $L(t)$ at high levels, as well as the change in the value of $L(t)$ for an instantaneous pressure of $0 \mathrm{~Pa}$, agree qualitatively with most published recordings of inner-hair-cell membrane potentials (e.g., Russell and Sellick, 1983, their Figs. 1, 2, 10B; Russell et al., 1986a, their Figs. 5A, 9, 10E; Russell et al., 1986b, their Fig. 3A; Dallos, 1985, his Fig. 5; Dallos, 1986, his Fig. 6; Dallos and Cheatham, 1989, their Fig. 1; Dallos and Cheatham, 1990, their Figs. 2, 3). With a cutoff frequency higher than the stimulus frequency, the model is also able to produce filter outputs that are less sinusoidal and contain distortions. The magnitude of the AC component of $L(t)$ typically grows linearly with increasing stimulus amplitude before saturating (not shown). This is consistent with the growth and saturation of the AC component of the receptor potential of inner hair cells (e.g., Russell and Sellick, 1978; Dallos, 1985; Patuzzi and Yates, 1987). The magnitude of the DC component of the change in $L(t)$ (defined as the mean value minus the resting value) typically grows (but, in cases where $M_{0}>0.5$, decreases) with the square of the stimulus amplitude before saturating (not shown). This is consistent with the often steeper-than-linear growth and saturation of the DC component of the receptor potential of inner hair cells (Patuzzi and Sellick, 1983; Dallos, 1985). The AC/DC ratio of the change in $L(t)$ therefore decreases linearly with increasing stimulus amplitude before saturating, or, equivalently, decreases with the square root of the DC component. This is consistent with experimental findings (see Patuzzi and Sellick, 1983, their Fig. 7). These behaviors of the AC and DC components in the model result from the Boltzmann transducer function and are not qualitatively affected by the filter.

Many period histograms computed from ANF responses to tones can be well approximated by a sinusoid passed through an exponential transfer function. Because $L(t)$ in the model presented here is approximately sinusoidal (Fig. $1 E$ ), it is necessary to include an exponential transfer function, as follows:

$$
R(t)=C \cdot \mathrm{e}^{D \cdot L(t)}
$$

to relate the instantaneous output of the lowpass filter to the instantaneous rate of release events. Here, $C(>0)$ is the scale factor that specifies the rate (in events/s) for $L=0$, and $D(>0)$ is the slope factor. To keep the number of model parameters small, $C$ was fixed such that the model necessarily reproduced the spontaneous event rate $R_{\text {spont }}$. During spontaneous activity, $R(t)$ in Equation 8 is equal to $R_{\text {spont }}$, and the lowpass filter output $L(t)$ is equal to the normalized MET current at rest, $M_{0}$, such that $C=R_{\text {spont }} \cdot e^{-D \cdot M_{0}}$. This allows Equation 8 to be rewritten as follows:

$$
R(t)=R_{\text {spont }} \cdot e^{D \cdot\left(L(t)-M_{0}\right)} .
$$

Figure $1 F$ shows this exponential transfer function. Fixing $C$ also ensured that the model would fit period histograms in which the rate was modulated near the SR (i.e., near threshold), regardless of whether such histograms met the quality criteria to be included in the fits.

For simplicity, we assume that ANF spontaneous activity is described by a dead-time-modified homogeneous Poisson process (although the spiking statistics deviate somewhat from this assumption; see, e.g., Peterson and Heil, 2018). For such a process, the mean interspike interval equals the sum of the mean interevent interval and the mean refractory period (Young and Barta, 1986). The spontaneous event rate is therefore given by the inverse of the difference between the mean interspike interval during spontaneous activity and the mean refractory period. Here, we assumed a mean refractory period of $1.2 \mathrm{~ms}$ (Heil et al., 2007; Peterson et al., 2014, 2018; Peterson and Heil, 2018). Figure $1 G$ shows the instantaneous event rates obtained by passing the filter outputs in Figure $1 E$ through the exponential function in Figure $1 F$.

Figure $1 H$ shows the model's overall transfer functions relating the instantaneous stimulus pressure (Fig. $1 A$ ) to the instantaneous event rate (Fig. $1 G$ ). They are approximately exponential and have level-dependent slopes and operating points, despite the model having no level-dependent parameters. If these overall transfer functions were perfectly exponential, they would exactly correspond to the single, level-dependent exponential transfer step (Eq. 4) in the model of Peterson and Heil (2019), which relates their mechanical drive to the rate of release events.

\section{Model fits to exemplary data}

The current model was fitted to the same data as used in Peterson and Heil (2019), excluding six level series having fewer than five stimulus levels. The data comprise period histograms from 178 level series recorded from 77 ANFs. Figures 2-5 compare fit results obtained from the current model with those obtained by Peterson and Heil (2019) for four example ANFs having mid and high (relative to the phase-locking range of up to $\sim 5 \mathrm{kHz}$ in cat) characteristic frequencies (CFs) and low and high SRs. Figure 2 shows data for a level series of a representative mid-CF $(1.3 \mathrm{kHz})$, high-SR (62 spikes/s) ANF. Figure $2 A$ shows the period histograms of event rates (colored by stimulus level) and corresponding fits from the current model (white lines), whose four independent parameters $\left(M_{0}, b, f_{c}\right.$, and $\left.D\right)$ are constant across all period histograms shown. The maximum instantaneous event rate reached for each stimulus level (i.e., the rate at the peak of the period histogram) increases with increasing stimulus level before saturating, a finding common to all ANFs. In this example, the minimum instantaneous event rate (i.e., the rate in the trough of the period histogram) decreases with increasing stimulus level. This can be seen by comparing the rate in the troughs with the spontaneous event rate marked by the horizontal dotted line. Across the population of ANFs, both increases and decreases in the minimum instantaneous event rate with increasing stimulus level are observed (addressed below in The dependence of the minimum rate on level, frequency, and $\mathrm{SR}$ ). Figure $2 B$ shows the overall transfer functions (i.e., the functions relating the instantaneous stimulus pressure to the instantaneous rate of release events) obtained from these fits. They are approximately exponential (and therefore form approximately straight lines in the logarithmic plot used here) and their slopes decrease with increasing stimulus level. We assume the period histograms follow the von Mises distribution (i.e., one cycle of a sinusoid passed through an exponential function) (Ashida et al., 2010, 2013; van Hemmen, 2013; Peterson and Heil, 2019). In this case, a slope factor $B$ and scale factor $A$ can be computed for each overall transfer function (denoted overall $B$ and overall $A$ ) from the model's vector strength $V$ and mean rate $R_{\text {mean }}$ using the relationship as follows:

$$
V=\frac{I_{1}\left(B P_{1}\right)}{I_{0}\left(B P_{1}\right)}=\frac{I_{1}\left(B P_{1}\right) \cdot \mathrm{A}}{R_{\text {mean }}}
$$

where $I_{0}$ and $I_{1}$ are the zero-order and first-order modified Bessel functions of the first kind (Ashida et al., 2013; van 

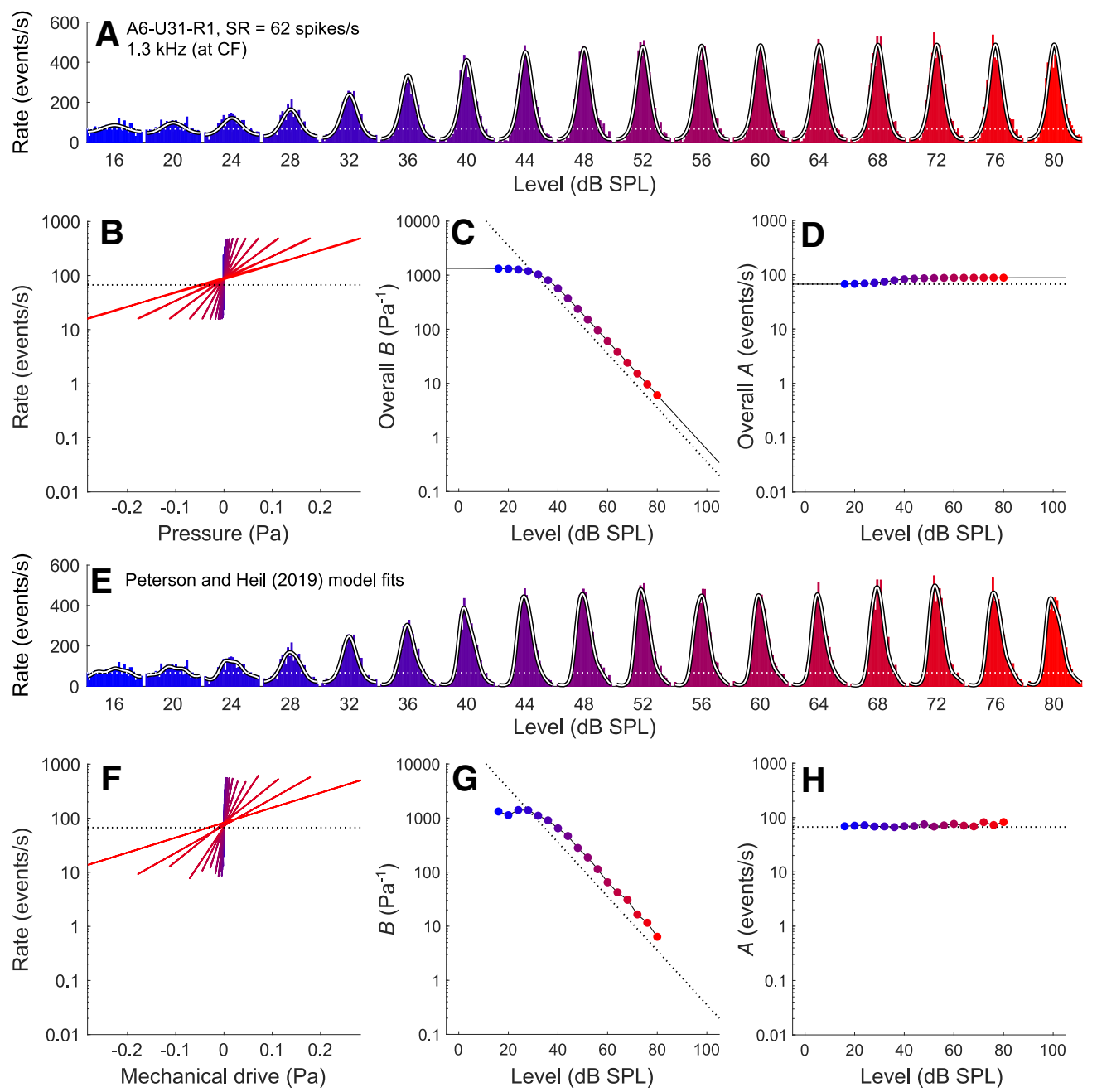

Figure 2. Comparison of fit results obtained from the current model and those obtained by Peterson and Heil (2019) for a representative mid-CF, high-SR ANF (A6-U31-R1; CF =1.3 kHz; $S R=62$ spikes/s) in response to (F tones from 16 to $80 \mathrm{~dB}$ SPL. A, Period histograms of event rates (colored by stimulus level) and corresponding fits from the current model (white lines), which uses only four independent parameters to fit all period histograms for a given ANF and stimulus frequency. The model reproduces the period histograms well. The best-fitting parameters for this example are $M_{0}=0.45, b=2006.64 \mathrm{~Pa}^{-1}, f_{\mathrm{c}}=1.07 \mathrm{kHz}$, and $D=5.48$. $B$, The overall transfer functions relating the instantaneous stimulus pressure to the instantaneous rate of release events obtained from these fits are approximately exponential and change with level. $\boldsymbol{C}$, The slope factor (overall $B$ ) as a function of the stimulus level. $\boldsymbol{D}$, The scale factor (overall $A$ ) as a function of the stimulus level. $A, B, D$, Horizontal dotted lines indicate the spontaneous event rate. $C$, Dotted negative diagonal indicates where the product of overall $B$ and the stimulus amplitude $P_{1}$ is equal to 1. C, D, Solid lines connecting the model data points also show extrapolations to lower and higher stimulus levels. $\boldsymbol{E}-\boldsymbol{H}$, Corresponding results from the Peterson and Heil (2019) model, which uses seven independent parameters to fit each period histogram.

Hemmen, 2013; Peterson and Heil, 2019). Figure 2C shows overall $B$ as a function of stimulus level. This slope factor is approximately constant at low levels and then decreases with increasing level with a slope of $\sim-1$ in this double-logarithmic plot. Figure $2 D$ shows overall $A$ as a function of stimulus level. This scale factor increases minimally before saturating in this example.

Figure $2 E-H$ shows corresponding results from the Peterson and Heil (2019) model. This model can reproduce the period histograms in more detail (Fig. $2 E$ ), but at the cost of using seven independent parameters $\left(P_{2}, P_{3}, \varphi_{1}, \varphi_{2}, \varphi_{3}, A\right.$, and $\left.B\right)$ for each period histogram. Even if it were simplified by omitting the distortions and by aligning the period histograms and stimulus waveforms as for the current model, there would still be two independent parameters ( $A$ and $B$ ) for each period histogram, corresponding to 34 independent parameters across the 17 histograms in this example. The exponential transfer functions (Fig. $2 F$ ) relating the instantaneous mechanical drive (Eq. 3) to the instantaneous rate of release events (Eq. 4) are similar to the overall transfer functions obtained with the current model (Fig. $2 B$ ), as are the $B$ versus level function (compare Fig. $2 G$ with
Fig. 2C) and the $A$ versus level function (compare Fig. $2 H$ with Fig. 2D).

Figure 3 shows data for a level series of a mid-CF $(1.2 \mathrm{kHz})$, low-SR (0.1 spikes/s) ANF. The fit results obtained from the current model are also very similar to those obtained with the model of Peterson and Heil (2019). In this example, in contrast to the previous one, the minimum instantaneous event rate reached for each stimulus level increases with increasing level before saturating (best seen in Fig. 3B,F). The minimum instantaneous event rate is higher than the spontaneous event rate (Fig. $3 B, F$, horizontal dotted lines) at all but the lowest stimulus levels. For both models, the $B$ versus level functions are approximately constant at low levels before decreasing with increasing level (Fig. 3C,G), and the $A$ versus level functions increase with increasing level before saturating (Fig. 3D,H).

Figure 4 shows data for a level series of a high-CF $(3.1 \mathrm{kHz})$, high-SR (51 spikes/s) ANF, and Figure 5 shows data for a level series of a high-CF $(4.1 \mathrm{kHz})$, low-SR (0.08 spikes/s) ANF. The data behave similarly to those of the mid-CF ANF in Figure 3, but the ratio of the maximum to the minimum instantaneous 

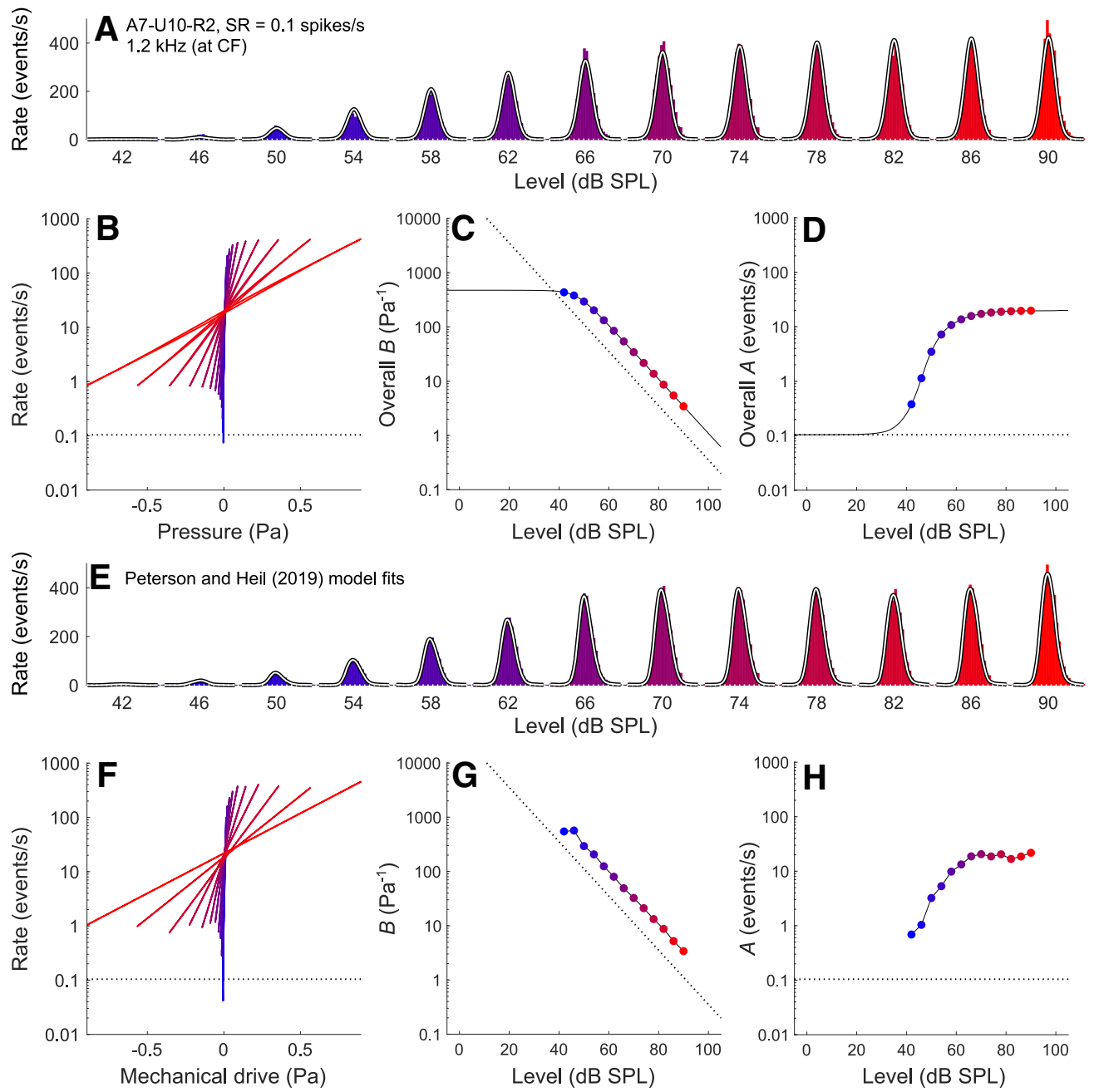

Figure 3. Comparison of fit results obtained from the current model and those obtained by Peterson and Heil (2019) for a representative mid-CF, low-SR ANF (A7-U10-R2; CF =1.2 kHz; $S R=0.1$ spikes/s) in response to (F tones from 42 to $90 \mathrm{~dB}$ SPL. $A$, Period histograms of event rates (colored by stimulus level) and corresponding fits from the current model (white lines). The best-fitting parameters for the current model are $M_{0}=0.25, b=512.72 \mathrm{~Pa}^{-1}, f_{\mathrm{c}}=0.75 \mathrm{kHz}$, and $D=20.89$. $\boldsymbol{B}$, The overall transfer functions relating the instantaneous stimulus pressure to the instantaneous rate of release events. $\boldsymbol{C}$, The slope factor (overall $B$ ) as a function of the stimulus level. $\boldsymbol{D}$, The scale factor (overall $\boldsymbol{A}$ ) as a function of the stimulus level. $\boldsymbol{E}$ - $\boldsymbol{H}$, Corresponding results from the Peterson and Heil (2019) model.

event rates (and therefore the height of the functions in this logarithmic plot) is smaller for these high-CF ANFs due to the stronger attenuation of the AC component by lowpass filtering. For both examples, the fit results obtained from the current model are very similar to those obtained with the model of Peterson and Heil (2019).

\section{Population results}

Figures 6 and 7 show population results for parameter estimates from the current model.

\section{Parameters of the Boltzmann transducer function}

Figure $6 \mathrm{~A}$ shows the estimates of the normalized MET current at rest, $M_{0}$, as functions of stimulus frequency (horizontal axis) and spontaneous event rate (color code). The estimates vary between 0.05 and 0.70 , and their distribution is bimodal (Fig. $6 \mathrm{~A}$, inset). The peak at 0.05 results predominantly from low-SR ANFs and from a few high-SR ANFs studied with high-frequency tones, whereas the peak at 0.45 results predominantly from high-SR ANFs. Estimates of $M_{0}$ are positively correlated with spontaneous event rate (Spearman's rank correlation coefficient $\sigma=0.64$, $p=3.9 \times 10^{-22}, n=178$ level series) and negatively correlated with frequency $\left(\sigma=-0.49, p=3.3 \times 10^{-12}\right)$ and with CF $(\sigma=$ $\left.-0.52, p=1.6 \times 10^{-13}\right)$. However, for individual ANFs tested with multiple stimulus frequencies, there is no consistent change in $M_{0}$ with stimulus frequency (data not shown). To examine the robustness of the estimates of $M_{0}$, we compared the negative log likelihoods obtained from model fits having different values of $M_{0}$. For each level series, a relative measure of the quality of the fit was computed as the difference between the negative log likelihood obtained for each $M_{0}$ and the negative log likelihood obtained for the best $M_{0}$, such that the smaller the difference, the better the fit. Figure $6 \mathrm{~B}$ shows this measure as a function of $M_{0}$ for each level series (connected by lines, where color represents stimulus frequency). The three subpanels show results grouped by SR (Fig. 6 Bi-Biii correspond to $>10$ spikes/s, 1-10 spikes/s, and $<1$ spike/s, respectively). Functions obtained from high-SR ANFs using low stimulus frequencies are $\mathrm{V}$-shaped and show clear minima (Fig. 6Bi). Most functions obtained using high stimulus frequencies or obtained from intermediate- and low-SR ANFs (Fig. 6Bii,Biii) do not show clear minima. Instead, the quality of the fits is typically comparable for all values of $M_{0}$ between 0.05 and 0.45 , revealing that a range of values is compatible with these data. This implies that the distribution of the true values of $M_{0}$ for a given frequency could be narrower than that of the estimated values. The fits performed on simulated period histograms generated with known model parameters (to verify 

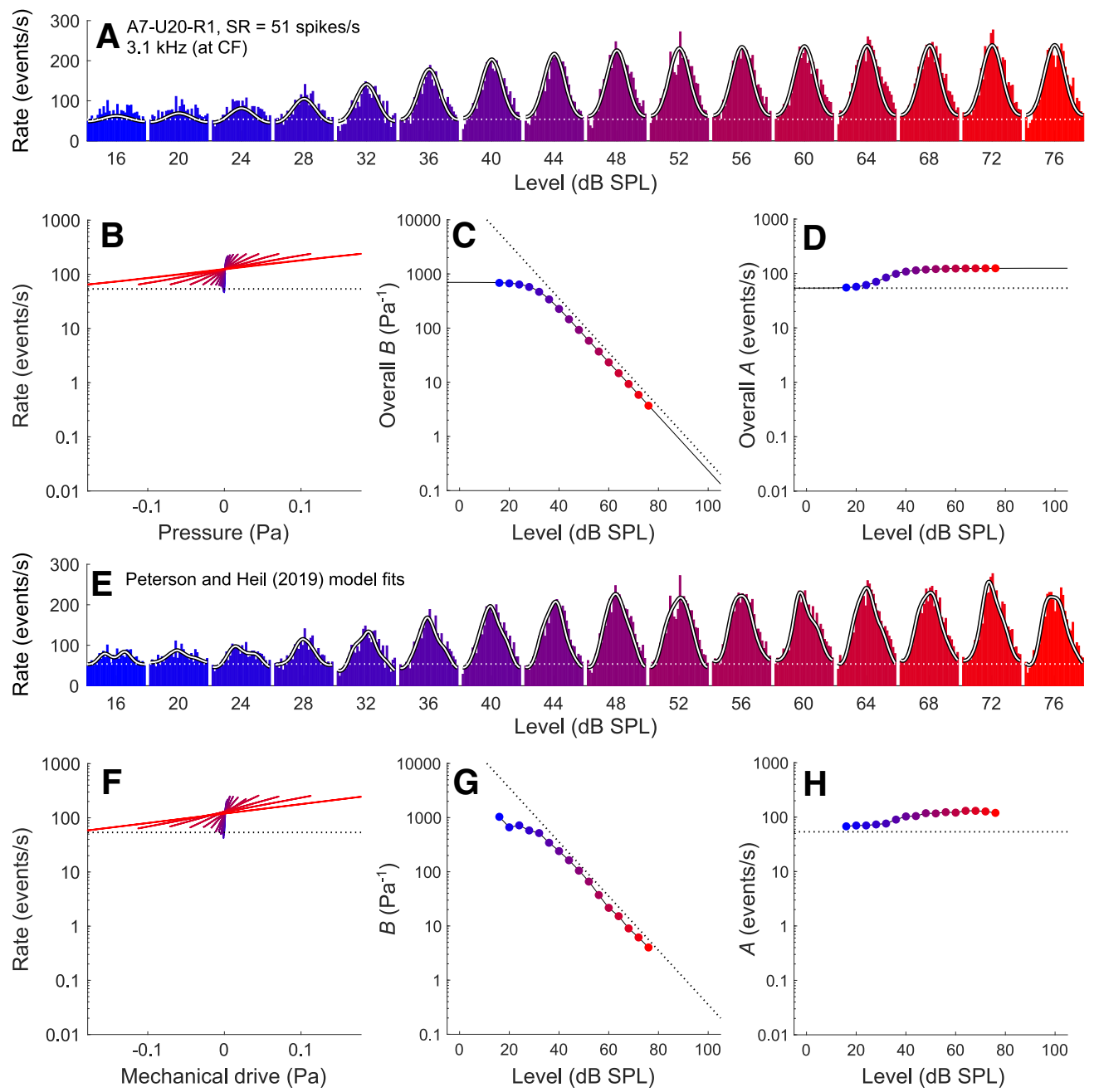

Figure 4. Comparison of fit results obtained from the current model and those obtained by Peterson and Heil (2019) for a representative high-CF, high-SR ANF (A7-U20-R1; CF =3.1 kHz; $\mathrm{SR}=51$ spikes/s) in response to (F tones from 16 to $76 \mathrm{~dB}$ SPL. $A$, Period histograms of event rates (colored by stimulus level) and corresponding fits from the current model (white lines). . The best-fitting parameters for the current model are $M_{0}=0.40, b=2782.56 \mathrm{~Pa}^{-1}, f_{\mathrm{c}}=1.54 \mathrm{kHz}$, and $D=8.41$. $\boldsymbol{B}$, The overall transfer functions relating the instantaneous stimulus pressure to the instantaneous rate of release events. $\boldsymbol{C}$, The slope factor (overall $B$ ) as a function of the stimulus level. $\boldsymbol{D}$, The scale factor (overall $A$ ) as a function of the stimulus level. $\boldsymbol{E}$ - $\boldsymbol{H}$, Corresponding results from the Peterson and Heil (2019) model.

the reliability of the grid-based fitting procedure; see Materials and Methods) yielded functions very similar to those obtained for the real period histograms, including those having flat portions for $M_{0} \leq 0.45$.

Figure $6 C$ shows the estimates of the slope factor $b$ of the Boltzmann transducer function as functions of the lowest stimulus level included (horizontal axis) and of the spontaneous event rate (color code). The lowest stimulus level included can serve as a proxy for the threshold stimulus level of the ANF to the stimulus frequency and is therefore a measure of the inverse of the ANF's sensitivity to that frequency. Because $b$ acts as a scale factor for the stimulus pressure, it also reflects that sensitivity. The two measures should therefore be negatively correlated. Indeed, for a given SR, $b$ decreases as the lowest stimulus level increases. The slope is $\sim-1$ in this plot, such that the dimensionless products of $b\left(\right.$ in $\left.\mathrm{Pa}^{-1}\right)$ and the lowest stimulus amplitude (in $\mathrm{Pa}$ ) are approximately the same across ANFs of similar SR. For a given $b$, the lowest stimulus level is $15-30 \mathrm{~dB}$ higher for low-SR ANFs than for high-SR ANFs, which reflects the fact that low-SR ANFs are less sensitive (for review, see Heil and Peterson, 2015). The value of the product of $b$ and the stimulus amplitude $P_{1}\left(b P_{1}\right)$ determines the amount of modulation of the output of the Boltzmann transducer function and is therefore related to the vector strength. Figure $6 D$ shows the vector strength, obtained from each period histogram in the data, plotted as functions of $b P_{1}$ (horizontal axis) and of the stimulus frequency (color code). For a first-order Boltzmann function, the width of the range over which the normalized MET current changes from $10 \%$ to $90 \%$ of the maximum is equal to $\sim 4.4 / b$. Unless the system at rest sits far outside this range, stimulus pressures yielding values of $b P_{1}$ up to 4 or 5 should therefore approximately cover the steep portion of the transducer function. Indeed, vector strength increases rapidly when $b P_{1}$ exceeds $\sim 0.1$ and reaches its maximum when $b P_{1}$ is $\sim 5$. For low-frequency stimuli, the vector strength tends to decrease with further increases in $b P_{1}$ due to an increase in the harmonic distortions (Peterson and Heil, 2019). As the stimulus frequency increases, the maximum vector strength reached decreases due to lowpass filtering (e.g., Palmer and Russell, 1986; Weiss and Rose, 1988).

There is a relatively weak negative correlation between estimates of $M_{0}$ and $b\left(\sigma=-0.29, p=1.04 \times 10^{-4}\right)$. It likely reflects a compensatory relationship between the two parameters. For a constant $b$, an increase or decrease in $M_{0}$ causes the MET current to saturate at a lower or higher stimulus level, respectively. To counter this effect, $b$ must decrease or increase, respectively. This 

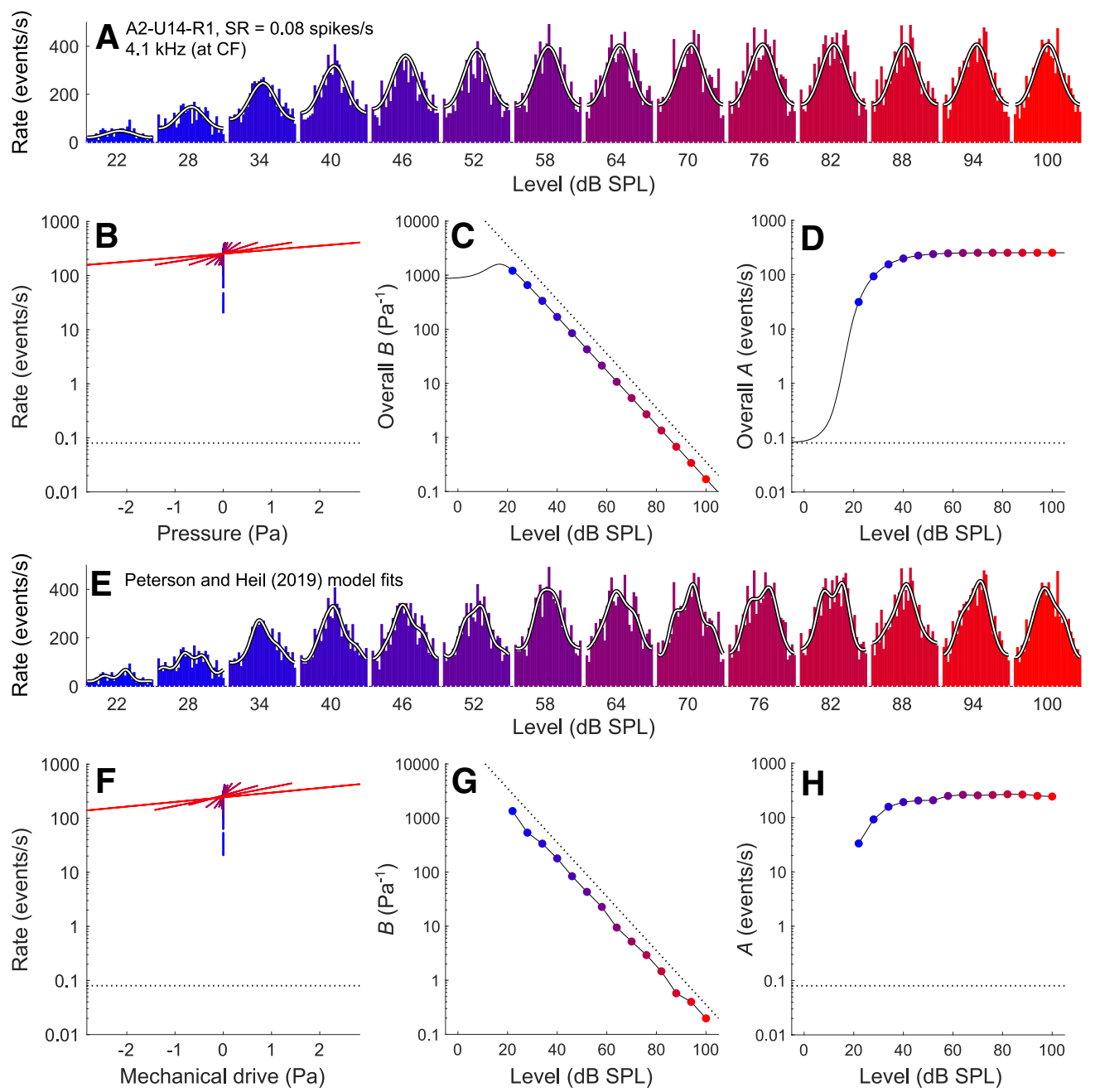

Figure 5. Comparison of fit results obtained from the current model and those obtained by Peterson and Heil (2019) for a representative high-CF, low-SR ANF (A2-U14-R1; CF = 4.1 kHz; $S R=0.08$ spikes/s) in response to (F tones from 22 to $100 \mathrm{~dB}$ SPL. A, Period histograms of event rates (colored by stimulus level) and corresponding fits from the current model (white lines). The best-fitting parameters for the current model are $M_{0}=0.05, b=23798.15 \mathrm{~Pa}^{-1}, f_{\mathrm{c}}=1.42 \mathrm{kHz}$, and $D=17.93$. $\boldsymbol{B}$, The overall transfer functions relating the instantaneous stimulus pressure to the instantaneous rate of release events. $\boldsymbol{C}$, The slope factor (overall $B$ ) as a function of the stimulus level. $\boldsymbol{D}$, The scale factor (overall $A$ ) as a function of the stimulus level. $\boldsymbol{E}$ - $\boldsymbol{H}$, Corresponding results from the Peterson and Heil (2019) model.

too implies that the distribution of the true values of $M_{0}$ for a given frequency could be narrower than that of the estimated values.

\section{Cutoff of the lowpass filter}

Figure $7 A$ shows the estimates of the cutoff frequency of the lowpass filter, $f_{c}$, as functions of the stimulus frequency $f_{1}$ (horizontal axis) and of the spontaneous event rate (color code). The data points fall into two distinct groups: one below and one above the diagonal. For both groups, the estimates of $f_{c}$ increase with increasing stimulus frequency. This was also observed for estimates from individuals ANFs tested with multiple stimulus frequencies (not shown). The increase has a slope of $\sim 1$ in this double-logarithmic plot up to a stimulus frequency of $\sim 1.5 \mathrm{kHz}$, and a slope $<1$ for higher stimulus frequencies. The maximum estimate of $f_{\mathrm{c}}$ is $3.8 \mathrm{kHz}$. For the group below the diagonal, where $f_{\mathrm{c}}<f_{1}$, the filter strongly attenuates the upper harmonic distortion components introduced by the saturating transducer function, such that the outputs of the lowpass filter are approximately sinusoidal. The period histograms of ANFs in this group are approximately temporally symmetric. All examples from Figures $2-5$ belong to this group, which contains both low- and high-SR
ANFs. For a given stimulus frequency, the estimates of $f_{\mathrm{c}}$ tend to be lower for low-SR ANFs than for high-SR ANFs. For the group above the diagonal, where $f_{c}>f_{1}$ (typically between $2 \times f_{1}$ and 3 $\times f_{1}$ ), the filter does not strongly attenuate the upper harmonic distortion components introduced by the saturating transducer function. The period histograms of level series in this group become temporally asymmetric as the stimulus level increases. This group contains mostly high-SR ANFs with CFs predominantly $<1 \mathrm{kHz}$. Figure $8 A, B$ shows two examples from this group. In Figure $8 A$, the period histograms at higher stimulus levels are characterized by shoulders on their trailing edges and are well described by the model. In Figure $8 B$, the period histograms at higher stimulus levels are characterized by peak splitting and are not well described by the model. To achieve satisfactory fits, additional sources of distortion would be required in the model. The group in Figure $7 A$ for which $f_{c}>f_{1}$ also contains data from some level series whose period histograms are nearly (but not quite) symmetric and are fitted nearly as well by a second set of parameter values within the region of parameter space having $f_{\mathrm{c}}<f_{1}$. Figure $8 C, D$ shows one such example. Figure $8 C$ shows the best fit with $f_{c}>f_{1}$, and Figure $8 D$ shows the best fit with $f_{\mathrm{c}}<f_{1}$. Although the model fits differ in shape, they hardly differ in quality. Figure $7 B$ shows, for the 

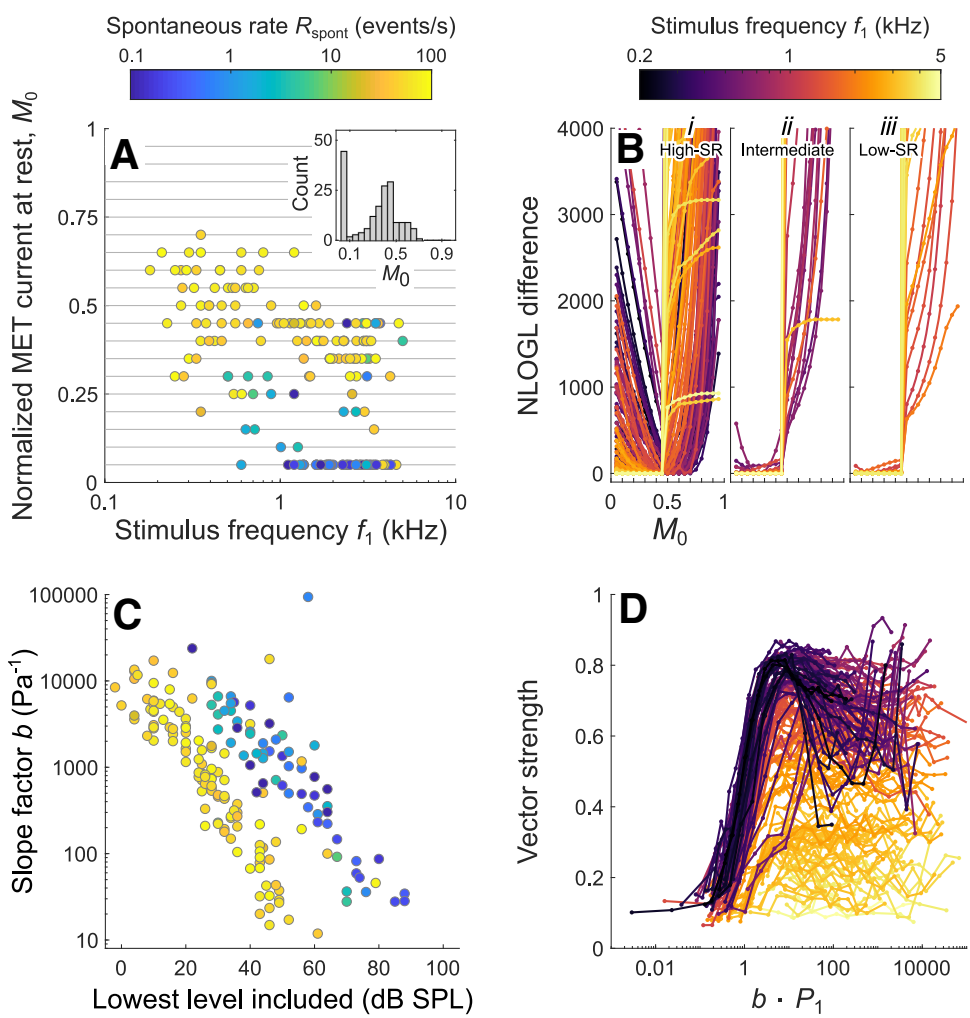

Figure 6. Parameter estimates for the Boltzmann transducer function from the current model. $\boldsymbol{A}$, Estimates of the normalized MET current at rest, $M_{0}$, plotted as a function of stimulus frequency and colored by spontaneous event rate. Inset, Distribution of $M_{0}$ estimates. $\boldsymbol{B}$, Difference between the negative log likelihood (NLOGL) for a given $M_{0}$ and the negative $\log$ likelihood for the best $M_{0}$, plotted as a function of $M_{0}$. Lines connect results for a given ANF and stimulus frequency and are colored by stimulus frequency. The three subpanels show results grouped by $\mathrm{SR}$ : $\boldsymbol{B i}-\mathbf{B i i i ,}>10$ spikes/s, 1-10 spikes/s, and $<1$ spike/s, respectively. $C$, Estimates of the slope factor $b$ of the Boltzmann transducer function, plotted as a function of the lowest stimulus level included in each fit and colored by spontaneous event rate. Stimulus levels were included only if they yielded $\geq 125$ spikes and significant vector strength. $\boldsymbol{D}$, Vector strength, obtained from each period histogram in the data, plotted as a function of the product of $b$ and the stimulus amplitude $P_{1}$. Lines connect results for a given ANF and stimulus frequency and are colored by stimulus frequency.

results in Figure $7 A$, the gain of each filter at the stimulus frequency. The gain was computed using Equation 7. Gains near 1 correspond to the group for which $f_{\mathrm{c}}>f_{1}$. All other gains correspond to the group for which $f_{\mathrm{c}}<f_{1}$. For this group, the gain is high $($ although $<1$ ) for low stimulus frequencies and then drops off steeply. The drop-off occurs at a slightly higher frequency and is slightly steeper for high-SR ANFs than for low-SR ANFs. Because low- and high-SR ANFs can innervate the same inner hair cells (Liberman, 1982; Wu et al., 2016), this implies a synaptic contribution to lowpass filtering that varies with the SR.

\section{Slope of the exponential transfer function}

Figure $7 C$ shows the estimates of the slope factor $D$ of the exponential transfer function plotted as a function of spontaneous event rate $R_{\text {spont }}$. Because $R_{\text {spont }}$ is proportional to $e^{D \cdot M_{0}}$, a given $R_{\text {spont }}$ can be reproduced by infinitely many combinations of $D$ and $M_{0}$. Nonetheless, estimates of $D$ are negatively correlated with $R_{\text {spont }}\left(\sigma=-0.65, p=4.9 \times 10^{-23}\right)$, and estimates of $M_{0}$ are positively correlated with $R_{\text {spont }}$ (see above), implying that the particular parameter combinations obtained from the fits are determined by the shapes of the period histograms. Estimates of $D$ are also positively correlated with stimulus frequency $(\sigma=0.41$, $\left.p=1.1 \times 10^{-8}\right)$ and with CF $\left(\sigma=0.41, p=1.4 \times 10^{-8}\right)$. These correlations are likely due to the absence of low-CF, low-SR ANFs in our sample. Estimates of $D$ are negatively correlated with estimates of the cutoff frequency $f_{\mathrm{c}}(\sigma=-0.37, p=3.9 \times$ $\left.10^{-7}\right)$. At least some of this correlation is likely due to parameter compensation. If the input to the lowpass filter were a sinusoid, then $D \times L(t)$ in Equation 8 would equal $D \times G \times P(t)$, where $G$ is the gain of the filter in Equation 7. Differences in the gain of the output due to differences in the cutoff frequency could then be perfectly compensated by changes in the slope factor $D$. This compensation is given by $D_{2} / D_{1}=G_{1} / G_{2}=$ $\sqrt{\left(1+\left(f / f_{c 2}\right)^{2 n}\right) /\left(1+\left(f / f_{c 1}\right)^{2 n}\right)}$. In the model, the input to the lowpass filter is not a sinusoid, but a sinusoid passed through a Boltzmann function, such that the compensation is therefore imperfect.

\section{Effects of constraining $\mathrm{M}_{0}$}

Low- and high-SR ANFs can innervate the same inner hair cells (Liberman, 1982; Wu et al., 2016). A single resting open probability of the MET channels (i.e., a single normalized MET current at rest, $M_{0}$ ) should therefore be able to account for the data of both low- and high-SR ANFs of similar CF. Given our observation that most of the level series best fitted by a value of $M_{0} \leq 0.45$ were similarly well fitted by all other values of $M_{0} \leq 0.45$ (Fig. $6 B$ ), the minimum value of $M_{0}$ can be constrained to any value $>$ 0.05 and $\leq 0.45$ without substantially affecting the quality of the fits. We examined how constraining $M_{0}$ to be $\geq 0.3$ affected the parameter estimates. This constraint predominantly affected estimates for low-SR ANFs. Nearly all values of $M_{0}$ originally $<0.3$ increased to 0.3 after imposing the constraint. This brings the estimates of $M_{0}$ for low- and high-SR ANFs having similar CF closer together in value, but not close enough to be entirely consistent with the expectation of very similar estimates for such ANFs. The constraint resulted in slight reductions in the strengths of the correlations between $M_{0}$ and spontaneous event rate $(\sigma=0.56, p=4.2 \times$ $\left.10^{-16}\right)$, stimulus frequency $\left(\sigma=-0.44, p=1.1 \times 10^{-9}\right)$, and $\mathrm{CF}$ $\left(\sigma=-0.45, p=1.7 \times 10^{-10}\right)$. It also affected the estimates of $f_{\mathrm{c}}$ (and therefore the gain) and $D$, which are shown in Figure $7 D-F$. For the low-SR ANFs affected, $f_{\mathrm{c}}$ and the gain decreased, leading to increased separations of $f_{\mathrm{c}}$ and gain values by spontaneous event rate (compare Fig. $7 D$ and Fig. $7 A$; and compare Fig. $7 E$ and Fig. $7 B$ ). If the imposed constraint is valid, then the synaptic contribution to lowpass filtering that varies with the SR might be much stronger than implied by Figure $7 A, B$. Finally, the strength of the negative correlation of $D$ with spontaneous event rate increased $\left(\sigma=-0.78, p=1.8 \times 10^{-37}\right.$; compare Fig. $7 F$ and Fig. $7 C)$. Parameter $D$ therefore likely reflects a property of the synapse that varies with the SR.

\section{The dependence of the minimum rate on level, frequency, and SR}

The maximum instantaneous event rate always increases with increasing stimulus level, whereas the minimum instantaneous event rate $R_{\min }$ can increase, remain constant, or decrease with increasing stimulus level (Figs. 2-5). Here, we examine whether these different behaviors of $R_{\min }$ depend on stimulus frequency 

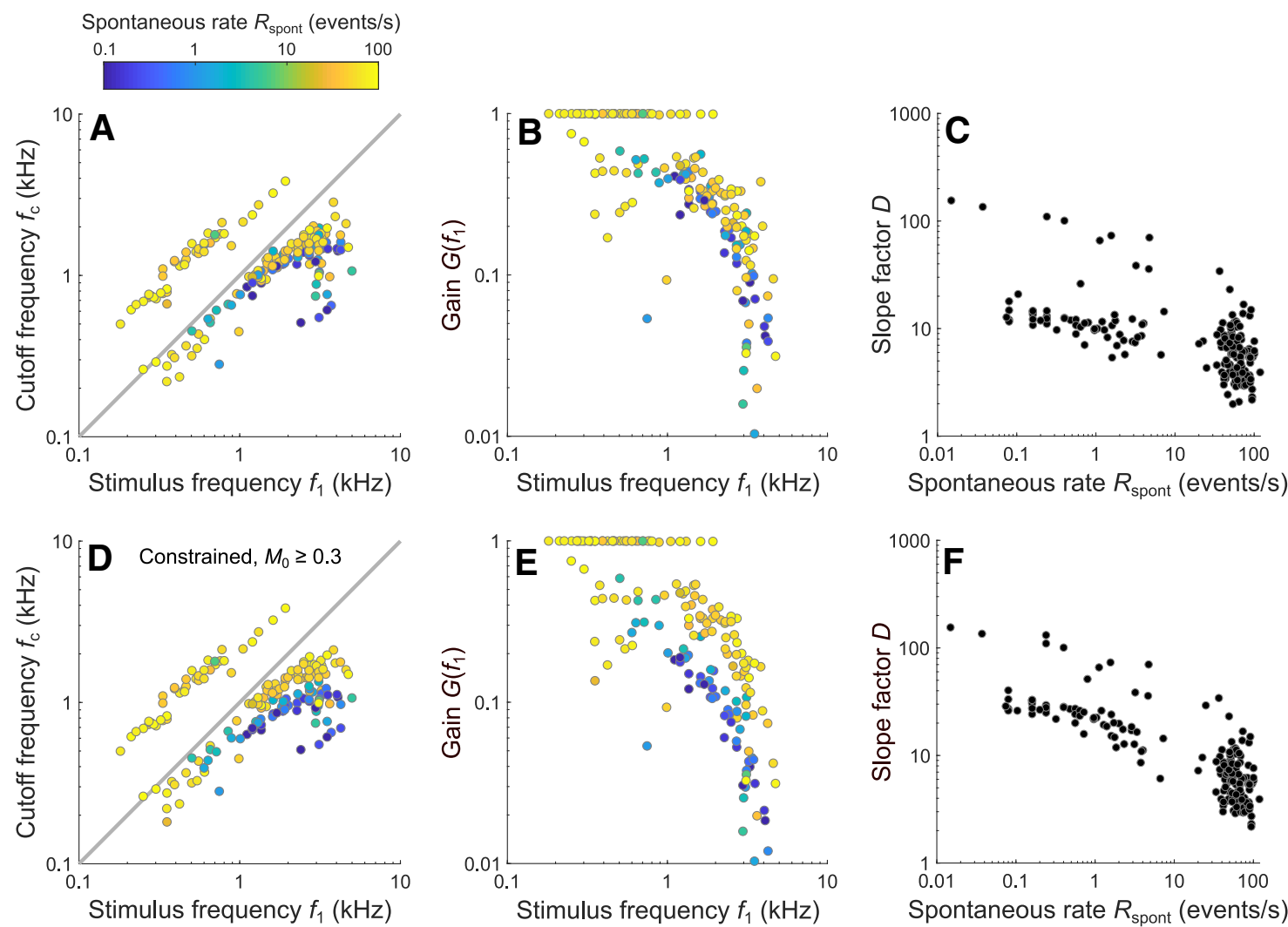

Figure 7. Parameter estimates for the lowpass filter and the exponential transfer function from the current model. $A$, Estimates of the cutoff frequency $f_{c}$ plotted as a function of the stimulus frequency $f_{1}$ and colored by spontaneous event rate. The data points fall into two groups characterized by $f_{\mathrm{c}}<f_{1}$ and $f_{\mathrm{c}}>f_{1}$. For all examples from Figures $2-5, f_{\mathrm{c}}<f_{1}$. B, Gain of each filter at the stimulus frequency, shown for the results in $\boldsymbol{A}$. $C$, Estimates of the slope factor $D$, plotted against the spontaneous event rate. $D-F$, Results corresponding to $A-C$ under the constraint that $M_{0} \geq 0.3$.

and SR, and how these behaviors can arise from the model. Figure $9 A-C, E, F, H, I$ shows the dependence of $R_{\min }$ on stimulus level for all ANFs. We used $R_{\min }$ obtained from the fitted model functions of Peterson and Heil (2019), which were found to describe the data closely, because $R_{\min }$ from the raw data itself would often equal zero due to the narrow histogram bins. Each panel shows $R_{\min }$ versus level functions grouped by ANF SR (where rows from top to bottom correspond to $>10$ spikes/s, 110 spikes/s, and $<1$ spike/s) and by stimulus frequency (where columns from left to right correspond to $<500 \mathrm{~Hz}, 500-2000 \mathrm{~Hz}$, and $>2000 \mathrm{~Hz}$ ). Gray lines connect adjacent stimulus levels, and the color of the symbols represents the vector strength. The $R_{\min }$ versus level functions marked by squares in Figure $9 B, H, C$, and $I$, respectively, correspond to the examples in Figures 2, 3, 4, and 5. For high-SR ANFs (Fig. $9 A-C$ ), $R_{\text {min }}$ predominantly decreases with increasing stimulus level. The decrease is most pronounced for low-frequency tones (Fig. 9A) and least pronounced for highfrequency tones (Fig. 9C). Some examples in Figure 9C show little change, or even slight increases, in $R_{\text {min }}$ with stimulus level. For intermediate-SR (Fig. 9E,F) and low-SR (Fig. 9H,I) ANFs, $R_{\text {min }}$ predominantly increases with increasing stimulus level. These results show that $R_{\min }$ versus level functions depend on both stimulus frequency and SR.

We performed simulations to explore the conditions under which $R_{\min }$ would increase, remain constant, or decrease with increasing stimulus level in the model. Because $R_{\min }$ is equal to the minimum output of the lowpass filter, $L_{\min }$, passed through the exponential transfer function, the direction of the change of $R_{\min }$ and $L_{\min }$ is the same. To avoid having to arbitrarily specify a value of the slope parameter of the exponential transfer function, we present the findings for $L_{\min }$ instead of for $R_{\min }$. Values of $L_{\text {min }}$ were calculated using a high stimulus level to ensure that saturation was reached. We found that whether $L_{\text {min }}$ (and therefore $R_{\min }$ ) increases or decreases depends on the cutoff frequency relative to the stimulus frequency, $f_{c} / f_{1}$, and on the normalized MET current at rest, $M_{0}$. Figure $9 D$ shows the difference between $L_{\min }$ and the lowpass filter output at rest, $L_{0}$ (identical in value to $M_{0}$ ), plotted as a function of $f_{\mathrm{c}} / f_{1}$ and $M_{0}$. A positive difference (where $L_{\min }-L_{0}>0$, marked red) corresponds to an increase in $R_{\min }$ with increasing stimulus level, whereas a negative difference (where $L_{\min }-L_{0}<0$, marked blue) corresponds to a decrease in $R_{\min }$. The value of $L_{\min }$ can increase above the resting value only when both $f_{\mathrm{c}}<f_{1}$ and $M_{0}$ $<0.5$ (red portion in lower left quadrant). For a given stimulus frequency, the observed transition of the $R_{\min }$ versus level functions from decreasing in high-SR ANFs (e.g., Fig. $9 B$ ) to increasing in low-SR ANFs (e.g., Fig. $9 H$ ) can only come about by decreasing $M_{0}$ or $f_{c}$. Low- and high-SR ANFs therefore must differ in $M_{0}$ or $f_{\mathrm{c}}$ or both. Because low- and high-SR ANFs innervating the same inner hair cells would "see" the same $M_{0}$, the SR dependence of the behavior of $R_{\min }$ is likely due to differences in $f_{\mathrm{c}}$ and therefore provides additional evidence for a synaptic contribution to lowpass filtering that varies with the SR.

Figure $9 G$ shows that the typical behaviors of low- and highSR ANFs can be reproduced, even when $M_{0}$ and $b$ are constant, as might be expected for a given inner hair cell and stimulus frequency. The period histograms simulated for a low-SR ANF (red curves) show an increase in $R_{\text {min }}$ with increasing stimulus level, whereas those simulated for a high-SR ANF (blue curves) show a decrease. Although both saturate at similar rates, the high-SR 

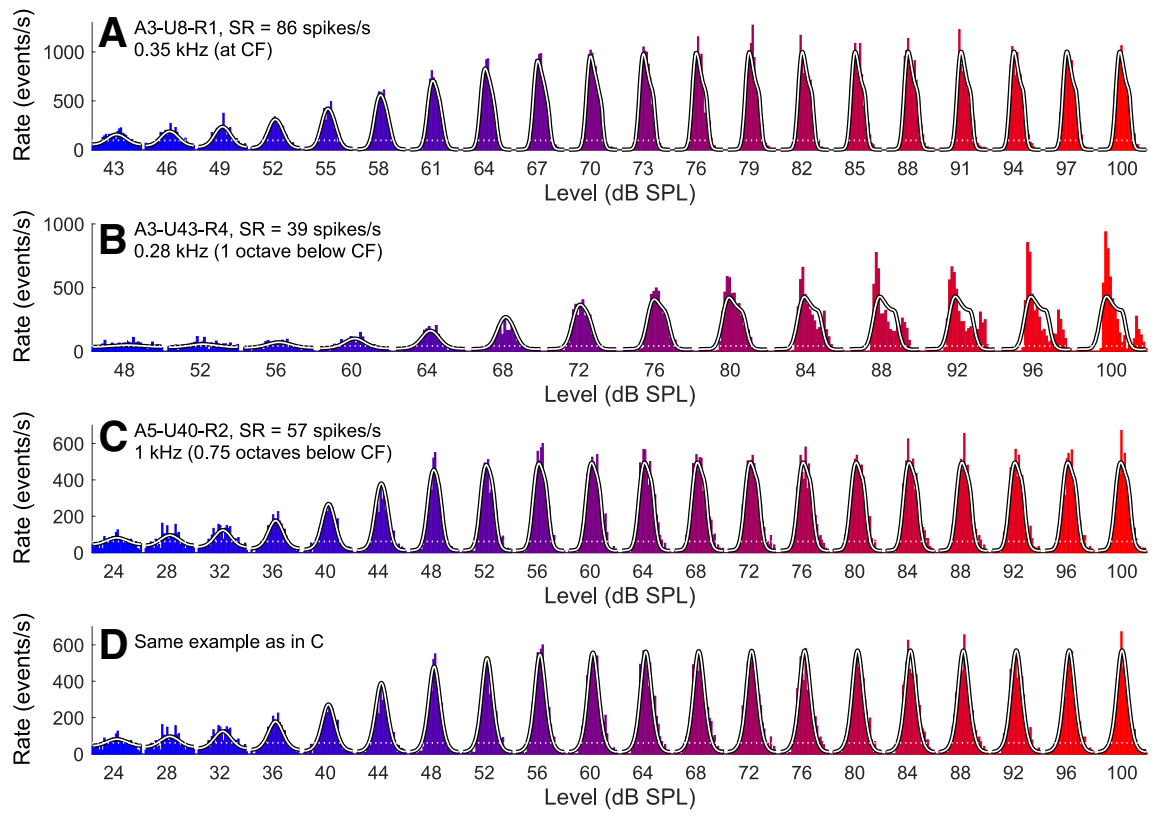

Figure 8. Period histograms and model fits of examples best fitted with a cutoff frequency higher than the stimulus frequency. $A$, Example (ANF A3-U8-R1; $C F=0.35 \mathrm{kHz} ; \mathrm{SR}=86 \mathrm{spikes} / \mathrm{s} ; f_{1}=0.35 \mathrm{kHz}$ ) characterized by temporally asymmetric period histograms without peak splitting. The best-fitting parameters are $M_{0}=0.65, b=105.85 \mathrm{~Pa}^{-1}, f_{\mathrm{c}}=0.82 \mathrm{kHz}$, and $D=5.46 . \quad B$, Example (ANF A3-U43-R4; $C F=0.55 \mathrm{kHz} ; \mathrm{SR}=39$ spikes $/ \mathrm{s} ; f_{1}=0.28 \mathrm{kHz}$ ) characterized by period histograms with peak splitting. The best-fitting parameters are $M_{0}=0.30, b=43.23 \mathrm{~Pa}^{-1}, f_{\mathrm{c}}=0.75 \mathrm{kHz}$, and $D=3.00$. C, $\boldsymbol{D}$, Example (ANF A5-U40-R2; (F $=1.8 \mathrm{kHz} ; \mathrm{SR}=57$ spikes $/ \mathrm{s} ; f_{1}=1.05 \mathrm{kHz}$ ) characterized by period histograms that are best fitted by a waveform having some distortions $\left(C ; M_{0}=0.45, b=774.26 \mathrm{~Pa}^{-1}, f_{\mathrm{c}}=2.15 \mathrm{kHz}\right.$, and $D=3.32$, with a negative log likelihood of $16,534.9)$ but which are fitted nearly as well with a cutoff frequency lower than the stimulus frequency $\left(\boldsymbol{D} ; M_{0}=0.45\right.$, $b=855.26 \mathrm{~Pa}^{-1}, f_{\mathrm{c}}=0.88 \mathrm{kHz}$, and $D=5.94$, with a negative log likelihood of $\left.16,570.5\right)$. Horizontal white dotted lines indicate the spontaneous event rates.

ANF reaches rate threshold and saturation at lower stimulus levels than does the low-SR ANF. These different behaviors are produced in the model by a higher $f_{\mathrm{c}}$ (resulting in a less attenuated input to the exponential transfer function) and a lower $D$ (i.e., a lower slope of the exponential transfer function) for the high-SR ANF than for the low-SR ANF. These parameter combinations cause opposite behaviors of $R_{\min }$ for low- and high-SR ANFs, yet result in similarly shaped period histograms at high stimulus levels (Fig. 9G).

\section{Discussion}

We have shown that the level-dependent exponential transfer functions reported by Peterson and Heil (2019) can be accounted for by a static Boltzmann transducer function that yields clipped MET currents at high stimulus levels, followed by a lowpass filter that transforms the currents back into approximately sinusoidal signals, followed by a static exponential transfer function that transforms the filter outputs into rates of synaptic release events (Fig. 1). Although the model is a highly simplified description of auditory peripheral processing, it is viable. For example, it reproduces the shape of the inner-hair-cell receptor potential and the changes of the AC and DC components with stimulus level. It removes the need for alternative mechanisms, such as level-dependent changes in the transduction machinery in the inner-hair-cell stereocilia. The model accounts for the level dependence of the operating point and slope of the exponential transfer function reported by Peterson and Heil (2019), for the rapidity of this apparent gain control, and for the level dependence of the maximum and minimum instantaneous spike rates (Figs. 2-9). The model does not reproduce histograms exhibiting strong distortions and peak splitting (Fig. $8 B$ ) or highly non- monotonic rate-level functions (e.g., Nelson's notch; for review, see Heil and Peterson, 2019).

\section{The Boltzmann function}

A first-order Boltzmann function was used to describe the instantaneous relationship between the stimulus pressure and the MET current. Fettiplace and Kim (2014) note that the second-order Boltzmann functions sometimes thought to describe the relationship between stereocilia displacement and the MET current in hair cells studied ex vivo might be a consequence of artificial mechanical stimulation (see also Corey et al., 2017). Although several models include a second-order Boltzmann function (Sumner et al., 2002; Zeddies and Siegel, 2004; Lopez-Poveda and Eustaquio-Martín, 2006; Altoè et al., 2018) or a similarly complicated logarithm-based function (Zhang et al., 2001; Zilany et al., 2009; Bruce et al., 2018), a first-order Boltzmann function (equivalent to a scaled and shifted tangent hyperbolic function) (Carney, 1993; Horst et al., 2018) sufficed for our purposes.

We found a bimodal distribution of the normalized MET current at rest, $M_{0}$ (equal to the model's resting open probability of the MET channels) (Fig. 6A). Notably, the data underlying the peak at 0.05 were fitted nearly equally well by a range of values of $M_{0}$ up to $\sim 0.45$ (Fig. $6 B$ ), meaning that the true values of $M_{0}$ might be higher than the estimates of 0.05 . It is unclear why, in these cases, the model fits tend to slightly favor $M_{0}=0.05$, but these low estimates are presumably unrealistic and might reflect compensation for incorrect assumptions about the filter properties or for some unknown element missing from the model. Identifying the true values of $M_{0}$ might require a more complete biophysical model that includes components such as voltage-dependent potassium channels, vesicle-pool dynamics, and perhaps even postsynaptic mechanisms.

We found a negative correlation between $M_{0}$ and CF (and between $M_{0}$ and the stimulus frequency, which was typically at or near CF), even when we constrained $M_{0}$ to $\geq 0.3$. Such a correlation is consistent with the findings of Johnson (2015) that resting MET currents are larger for apical than for basal inner hair cells and that the MET currents elicited by sinusoidal fluidjet stimulation of the hair bundles are more symmetrical about the resting current for apical than for basal inner hair cells (see Johnson, 2015, his Fig. 1). A decrease of $M_{0}$ from a value near 0.5 (the point of maximal sensitivity) with increasing CF or stimulus frequency would be useful because, as frequency increases, attenuation of the AC component of the inner-hair-cell membrane potential by lowpass filtering increases (Russell and Sellick, 1978; Patuzzi and Sellick, 1983; Palmer and Russell, 1986), and the presence of a DC component becomes increasingly relevant. Jia et al. (2007) obtained somewhat lower estimates of the resting open probability for apical and basal inner hair cells recorded in the gerbil hemicochlea (respectively, 0.137 and 0.129 in $50 \mu \mathrm{M}$ $\mathrm{Ca}^{2+}$ ). Johnson et al. (2011) obtained similarly low estimates 

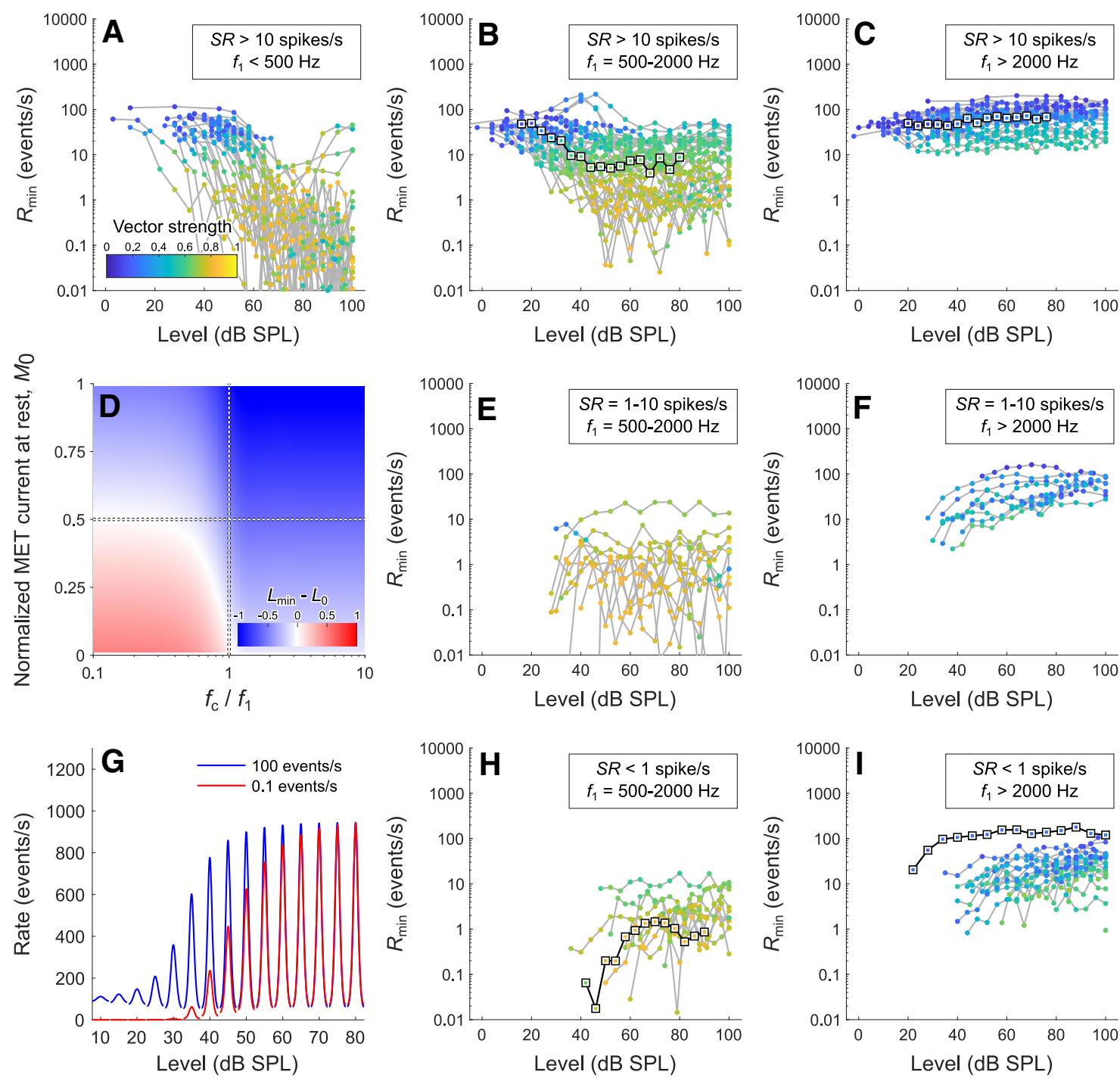

Figure 9. $A-C, E, F, H, I$, Dependence of the minimum instantaneous event rate $R_{\min }$ obtained from the fits of the model of Peterson and Heil (2019), on stimulus level, stimulus frequency, and SR. $R_{\min }$ versus level functions are grouped by SR (rows) and stimulus frequency (columns). Gray lines connect adjacent stimulus levels. Color of the symbols represents the vector strength for the stimulus frequency $f_{1}, \boldsymbol{B}, \boldsymbol{H}, \boldsymbol{C}, \boldsymbol{I}$, Example functions marked by squares represent the examples in Figures 2-5, respectively. $\boldsymbol{D}$, Differences between the minimum lowpass filter output, $L_{\min }$ (calculated for $b P_{1}=100,000$ ), and the lowpass filter output at rest, $L_{0}$, plotted as a function of $f_{c} / f_{1}$ and $M_{0}$. A positive difference (marked red) corresponds to an increase in $R_{\text {min }}$ with increasing stimulus level, whereas a negative difference (marked blue) corresponds to a decrease in $R_{\min }$. $G$, Typical behaviors of high- and low-SR ANFs can be reproduced by the model if the high-SR ANF has a higher $f_{\mathrm{c}}$ and a lower $D$ than the low-SR ANF, as demonstrated here using simulations with 100 and 0.1 events $/ \mathrm{s}$. For both SRs, $M_{0}=0.25$ and $b=3000 \mathrm{~Pa}{ }^{-1}$. For the high-SR ANF, $f_{\mathrm{c}} / f_{1}=0.9$ and $D=3.57$. For the low-SR ANF, $f_{\mathrm{c}} / f_{1}=0.4$ and $D=31.6$.

for gerbil apical inner hair cells in vitro $\left(0.17\right.$ in $\left.20 \mu \mathrm{M} \mathrm{Ca}{ }^{2+}\right)$. Such low values might not represent the in vivo values.

\section{The lowpass filter}

A third-order Butterworth lowpass filter was applied to the MET current. To keep the number of model parameters small, we used a single filter rather than several filters in series, although such a cascade might better describe the physiological processes in hair cells. We find that the cutoff frequencies fall into two groups characterized by $f_{\mathrm{c}}<f_{1}$ and $f_{\mathrm{c}}>f_{1}$. For period histograms lacking strong harmonic distortions, the model's cutoff frequency must be lower than the stimulus frequency to remove the distortions present in the clipped MET currents. Such period histograms are well described by the model (Figs. 2-5). Conversely, for period histograms containing clear harmonic distortions, the model's cutoff frequency typically exceeds the stimulus frequency. Histograms with mild distortions are also well described by the model (Fig. $8 A, C$ ), but those with very strong distortions or peak splitting are not (Fig. $8 B$ ), even when the cutoff frequency is high enough to pass the distortions present in the clipped MET currents. Such examples might require adding distortions to the input to reflect distortions already present in the forces deflecting inner-hair-cell stereocilia (e.g., Cody and Mountain, 1989; Kiang, 1990; Rhode and Cooper, 1996; Cheatham and Dallos, 1998; Cooper, 1998; Khanna and Hao, 1999; Mountain and Cody, 1999; Guinan, 2012).

Assuming that individual hair cells are isopotential, our finding that cutoff frequencies and gains tend to be lower for low-SR ANFs than for high-SR ANFs having similar CFs, particularly after constraining $M_{0}$ to be $\geq 0.3$ (Fig. $7 D, E$ ), implies that there is at least one additional filtering stage at the synapse whose cutoff frequency varies with SR. Such a stage is consistent with the SRdependent behavior of $R_{\min }$ (Fig. 9). Kidd and Weiss (1990) argue that the entry and accumulation of calcium at the synapse act as lowpass filters. Differences in the cutoff frequency for lowand high-SR ANFs might therefore come about by differences in the time constant of activation of the $\mathrm{Ca}^{2+}$ channels at the 
corresponding synapses. There is evidence that the voltage-gated $\mathrm{Ca}_{\mathrm{v}} 1.3$ channels that mediate synaptic transmission in inner hair cells (Platzer et al., 2000) are not all identical (Frank et al., 2009; Ohn et al., 2016; Vincent et al., 2017). Similarly, the amount of calcium present as a function of time, $x(t)$, given a rate of calcium clearance $\beta$, could equal the calcium current $\alpha(t)$ passed through a lowpass filter described by the differential equation $x^{\prime}(t)=\alpha(t)-\beta \cdot x(t)$ (Kidd and Weiss, 1990; Borst and Abarbanel, 2007). The cutoff frequency of such a filter is $f_{c}=\beta /(2 \pi)$. The lower cutoff frequencies we observed for low-SR ANFs might therefore come about by lower rates of calcium clearance. Differences in the rate of calcium clearance at individual synapses of a given inner hair cell might be mediated by differences in the concentrations or affinities of calcium buffers.

The lower the cutoff frequency, the smaller the AC/DC ratio of the filter output for stimulus frequency above the cutoff frequency. One might therefore expect vector strengths to be lower and to roll off earlier with increasing stimulus frequency for lowSR ANFs than for high-SR ANFs. However, vector strength tends to be higher for low-SR ANFs (Johnson, 1980; Joris et al., 1994; Louage et al., 2004; Dreyer and Delgutte, 2006; Temchin and Ruggero, 2010), and the rolloff is similar for low- and high-SR ANFs (Temchin and Ruggero, 2010, their Fig. 7A). The model is consistent with these findings because vector strength is not solely determined by the AC/DC ratio of the filter output; a decrease in the ratio can be compensated by an increase in the slope of the synaptic exponential transfer function. Very similar period histograms can therefore be obtained, even when cutoff frequencies differ (Fig. 9G, highest stimulus level).

The dependence of the cutoff frequency on the stimulus frequency obtained from the model fits (Fig. $7 A, D$ ) might be unexpected because the dependence of the AC/DC ratio of the membrane potential on stimulus frequency is typically interpreted as resulting from lowpass filters with fixed cutoff frequencies (e.g., Russell and Sellick, 1983; Palmer and Russell, 1986). If the model's cutoff frequency were fixed, however, then presenting sufficiently low stimulus frequencies would yield clipped period histograms inconsistent with the data. The dependence might reflect a previously unrecognized property of inner hair cells. Alternatively, other mechanisms would be needed to prevent peak clipping (e.g., gain control in the transduction machinery or perhaps mechanisms underlying spike-rate adaptation on time scales shorter than the stimulus period).

\section{The exponential transfer function}

An exponential transfer function was used to describe the instantaneous relationship between the filter output and the event rate. Given the tight relationship of the slope factor $D$ to the SR (Fig. $7), D$ likely reflects properties that vary with SR across synapses, such as the number of $\mathrm{Ca}_{V} 1.3$ channels (e.g., Frank et al., 2009; Meyer et al., 2009; Heil and Neubauer, 2010; Heil et al., 2011; Wong et al., 2013, 2014; Ohn et al., 2016; Peterson and Heil, 2018) and the voltage dependence of their gating (Ohn et al., 2016). The exponential function might reflect the lower exponential tail of this voltage dependence. Any power relating the synaptic $\mathrm{Ca}^{2+}$ signal and the rate of release events is contained within the scale and slope factors of the exponential function.

\section{References}

Altoè A, Pulkki V, Verhulst S (2018) The effects of the activation of the inner-hair-cell basolateral $\mathrm{K}^{+}$channels on auditory nerve responses. Hear Res 364:68-80.
Ashida G, Funabaki K, Carr CE (2013) Theoretical foundations of the sound analog membrane potential that underlies coincidence detection in the barn owl. Front Comput Neurosci 7:151.

Ashida G, Wagner H, Carr CE (2010) Processing of phase-locked spikes and periodic signals. In: Analysis of parallel spike trains (Grün S, Rotter S, eds), pp 59-74. New York: Springer.

Avissar M, Wittig JH Jr, Saunders JC, Parsons TD (2013) Refractoriness enhances temporal coding by auditory nerve fibers. J Neurosci 33:7681-7690.

Berry MJ 2nd, Meister M (1998) Refractoriness and neural precision. J Neurosci 18:2200-2211.

Borst A, Abarbanel HD (2007) Relating a calcium indicator signal to the unperturbed calcium concentration time-course. Theor Biol Med Model 4:7.

Bruce IC, Erfani Y, Zilany MS (2018) A phenomenological model of the synapse between the inner hair cell and auditory nerve: implications of limited neurotransmitter release sites. Hear Res 360:40-54.

Carney LH (1993) A model for the responses of low-frequency auditorynerve fibers in cat. J Acoust Soc Am 93:401-417.

Cheatham MA, Dallos P (1998) The level dependence of response phase: observations from cochlear hair cells. J Acoust Soc Am 104:356-369.

Cody AR, Mountain DC (1989) Low-frequency responses of inner hair cells: evidence for a mechanical origin of peak splitting. Hear Res 41:89-100.

Colburn HS (1973) Theory of binaural interaction based on auditory-nerve data: I. General strategy and preliminary results on interaural discrimination. J Acoust Soc Am 54:1458-1470.

Cooper NP (1998) Harmonic distortion on the basilar membrane in the basal turn of the guinea-pig cochlea. J Physiol 509:277-288.

Corey DP, Maoiléidigh DÓ, Ashmore JF (2017) Mechanical transduction processes in the hair cell. In: Understanding the cochlea: Springer handbook of auditory research (Manley GA, Gummer AW, Popper AN, Fay RR, eds), pp 75-111. New York: Springer.

Dallos P (1985) Response characteristics of mammalian cochlear hair cells. J Neurosci 5:1591-1608.

Dallos P (1986) Neurobiology of cochlear inner and outer hair cells: intracellular recordings. Hear Res 22:185-198.

Dallos P, Cheatham MA (1989) Nonlinearities in cochlear receptor potentials and their origins. J Acoust Soc Am 86:1790-1796.

Dallos P, Cheatham MA (1990) Effects of electrical polarization on inner hair cell receptor potentials. J Acoust Soc Am 87:1636-1647.

Dreyer A, Delgutte B (2006) Phase locking of auditory-nerve fibers to the envelopes of high-frequency sounds: implications for sound localization. J Neurophysiol 96:2327-2341.

Fettiplace R, Kim KX (2014) The physiology of mechanoelectrical transduction channels in hearing. Physiol Rev 94:951-986.

Frank T, Khimich D, Neef A, Moser T (2009) Mechanisms contributing to synaptic $\mathrm{Ca}^{2+}$ signals and their heterogeneity in hair cells. Proc Natl Acad Sci USA 106:4483-4488.

Grothe B, Pecka M, McAlpine D (2010) Mechanisms of sound localization in mammals. Physiol Rev 90:983-1012.

Guinan JJ (2012) How are inner hair cells stimulated? Evidence for multiple mechanical drives. Hear Res 292:35-50.

Heil P, Neubauer H (2010) Summing across different active zones can explain the quasi-linear $\mathrm{Ca}^{2+}$-dependencies of exocytosis by receptor cells. Front Synaptic Neurosci 2:148.

Heil P, Peterson AJ (2015) Basic response properties of auditory nerve fibers: a review. Cell Tissue Res 361:129-158.

Heil P, Peterson AJ (2017) Spike timing in auditory-nerve fibers during spontaneous activity and phase locking. Synapse 71:5-36.

Heil P, Peterson AJ (2019) Nelson's notch in the rate-level functions of auditory-nerve fibers might be caused by PIEZO2-mediated reverse-polarity currents in hair cells. Hear Res 381:107783.

Heil P, Neubauer H, Irvine DR, Brown M (2007) Spontaneous activity of auditory-nerve fibers: insights into stochastic processes at ribbon synapses. J Neurosci 27:8457-8474

Heil P, Neubauer H, Irvine DR (2011) An improved model for the rate: level functions of auditory-nerve fibers. J Neurosci 31:15424-15437.

Horst JW, McGee J, Walsh E (2018) Input-output curves of low and high spontaneous rate auditory nerve fibers are exponential near threshold. Hear Res 367:195-206.

Huet A, Desmadryl G, Justal T, Nouvian R, Puel JL, Bourien J (2018) The interplay between spike-time and spike-rate modes in the auditory nerve encodes tone-in-noise threshold. J Neurosci 38:5727-5738. 
Jia S, Dallos P, He DZ (2007) Mechanoelectric transduction of adult inner hair cells. J Neurosci 27:1006-1014.

Johnson DH (1974) The response of single auditory-nerve fibers in the cat to single tones: synchrony and average discharge rate. $\mathrm{PhD}$ Thesis, Department of Electrical Engineering, Massachusetts Institute of Technology, Cambridge, MA.

Johnson DH (1980) The relationship between spike rate and synchrony in responses of auditory-nerve fibers to single tones. J Acoust Soc Am 68:1115-1122.

Johnson DH, Swami A (1983) The transmission of signals by auditory-nerve fiber discharge patterns. J Acoust Soc Am 74:493-501.

Johnson SL (2015) Membrane properties specialize mammalian inner hair cells for frequency or intensity encoding. eLife 4:e08177.

Johnson SL, Beurg M, Marcotti W, Fettiplace R (2011) Prestin-driven cochlear amplification is not limited by the outer hair cell membrane time constant. Neuron 70:1143-1154.

Joris PX, Carney LH, Smith PH, Yin TC (1994) Enhancement of neural synchronization in the anteroventral cochlear nucleus: I. Responses to tones at the characteristic frequency. J Neurophysiol 71:1022-1036.

Joris PX, Schreiner CE, Rees A (2004) Neural processing of amplitude-modulated sounds. Physiol Rev 84:541-577.

Khanna SM, Hao LF (1999) Nonlinearity in the apical turn of living guinea pig cochlea. Hear Res 135:89-104.

Kiang NY (1990) Curious oddments of auditory-nerve studies. Hear Res 49:1-16.

Kidd RC, Weiss TF (1990) Mechanisms that degrade timing information in the cochlea. Hear Res 49:181-208.

Liberman MC (1982) Single-neuron labeling in the cat auditory nerve. Science 216:1239-1241.

Lopez-Poveda EA, Eustaquio-Martín A (2006) A biophysical model of the inner hair cell: the contribution of potassium currents to peripheral auditory compression. J Assoc Res Otolaryngol 7:218-235.

Lorenzi C, Gilbert G, Carn H, Garnier S, Moore BC (2006) Speech perception problems of the hearing impaired reflect inability to use temporal fine structure. Proc Natl Acad Sci USA 103:18866-18869.

Louage DH, van der Heijden M, Joris PX (2004) Temporal properties of responses to broadband noise in the auditory nerve. J Neurophysiol 91:2051-2065.

Meddis R (2006) Auditory-nerve first-spike latency and auditory absolute threshold: a computer model. J Acoust Soc Am 119:406-417.

Meenderink SW, van der Heijden M (2011) Dynamic aspects of cochlear microphonic potentials. AIP Conference Proceedings 1403:53-58.

Meyer AC, Frank T, Khimich D, Hoch G, Riedel D, Chapochnikov NM, Yarin YM, Harke B, Hell SW, Egner A, Moser T (2009) Tuning of synapse number, structure and function in the cochlea. Nat Neurosci 12:444-453.

Moore BC (2008) The role of temporal fine structure processing in pitch perception, masking, and speech perception for normal-hearing and hearing-impaired people. J Assoc Res Otolaryngol 9:399-406.

Mountain DC, Cody AR (1999) Multiple modes of inner hair cell stimulation. Hear Res 132:1-14.

Ohn TL, Rutherford MA, Jing Z, Jung S, Duque-Afonso CJ, Hoch G, Picher MM, Scharinger A, Strenzke N, Moser T (2016) Hair cells use active zones with different voltage dependence of $\mathrm{Ca}^{2+}$ influx to decompose sounds into complementary neural codes. Proc Natl Acad Sci USA 113: E4716-E4725

Palmer AR, Russell IJ (1986) Phase-locking in the cochlear nerve of the guinea-pig and its relation to the receptor potential of inner hair-cells. Hear Res 24:1-15.

Patuzzi R, Moleirinho A (1998) Automatic monitoring of mechano-electrical transduction in the guinea pig cochlea. Hear Res 125:1-16.

Patuzzi R, Sellick PM (1983) A comparison between basilar membrane and inner hair cell receptor potential input-output functions in the guinea pig cochlea. J Acoust Soc Am 74:1734-1741.

Patuzzi RB, Yates GK (1987) The low-frequency response of inner hair cells in the guinea pig cochlea: implications for fluid coupling and resonance of the stereocilia. Hear Res 30:83-98.

Peterson AJ (2020) MATLAB code for a model of phase locking with a lowpass filter. GitHub repository. Available at https://github.com/PetersonHeil/ MATLAB-code-for-a-model-of-phase-locking-with-a-lowpass-filter.
Peterson AJ, Heil P (2018) A simple model of the inner-hair-cell ribbon synapse accounts for mammalian auditory-nerve-fiber spontaneous spike times. Hear Res 363:1-27.

Peterson AJ, Heil P (2019) Phase locking of auditory-nerve fibers reveals stereotyped distortions and an exponential transfer function with a level-dependent slope. J Neurosci 39:4077-4099.

Peterson AJ, Irvine DR, Heil P (2014) A model of synaptic vesicle-pool depletion and replenishment can account for the interspike interval distributions and nonrenewal properties of spontaneous spike trains of auditorynerve fibers. J Neurosci 34:15097-15109.

Peterson AJ, Huet A, Bourien J, Puel JL, Heil P (2018) Recovery of auditorynerve-fiber spike amplitude under natural excitation conditions. Hear Res 370:248-263.

Platzer J, Engel J, Schrott-Fischer A, Stephan K, Bova S, Chen H, Zheng H, Striessnig J (2000) Congenital deafness and sinoatrial node dysfunction in mice lacking class D L-type $\mathrm{Ca}^{2+}$ channels. Cell 102:89-97.

Rhode WS, Cooper NP (1996) Nonlinear mechanics in the apical turn of the chinchilla cochlea in vivo. Aud Neurosci 2:101-121.

Robles L, Ruggero MA (2001) Mechanics of the mammalian cochlea. Physiol Rev 81:1305-1352.

Russell IJ, Sellick PM (1978) Intracellular studies of hair cells in the mammalian cochlea. J Physiol 284:261-290.

Russell IJ, Sellick PM (1983) Low-frequency characteristics of intracellularly recorded receptor potentials in guinea-pig cochlear hair cells. J Physiol 338:179-206.

Russell IJ, Cody AR, Richardson GP (1986a) The responses of inner and outer hair cells in the basal turn of the guinea-pig cochlea and in the mouse cochlea grown in vitro. Hear Res 22:199-216.

Russell IJ, Richardson GP, Cody AR (1986b) Mechanosensitivity of mammalian auditory hair cells in vitro. Nature 321:517-519.

Sumner CJ, Lopez-Poveda EA, O’Mard LP, Meddis R (2002) A revised model of the inner-hair cell and auditory-nerve complex. J Acoust Soc Am 111:2178-2188.

Temchin AN, Ruggero MA (2010) Phase-locked responses to tones of chinchilla auditory nerve fibers: implications for apical cochlear mechanics. J Assoc Res Otolaryngol 11:297-318.

van Hemmen JL (2013) Vector strength after Goldberg, Brown, and von Mises: biological and mathematical perspectives. Biol Cybern 107:385-396.

Verschooten E, Shamma S, Oxenham AJ, Moore BC, Joris PX, Heinz MG, Plack CJ (2019) The upper frequency limit for the use of phase locking to code temporal fine structure in humans: a compilation of viewpoints. Hear Res 377:109-121.

Vincent PF, Bouleau Y, Charpentier G, Emptoz A, Safieddine S, Petit C, Dulon D (2017) Different $\mathrm{Ca}_{\mathrm{v}} 1.3$ channel isoforms control distinct components of the synaptic vesicle cycle in auditory inner hair cells. J Neurosci 37:2960-2975.

Weiss TF, Rose C (1988) A comparison of synchronization filters in different auditory receptor organs. Hear Res 33:175-180.

Wong AB, Jing Z, Rutherford MA, Frank T, Strenzke N, Moser T (2013) Concurrent maturation of inner hair cell synaptic $\mathrm{Ca}^{2+}$ influx and auditory nerve spontaneous activity around hearing onset in mice. J Neurosci 33:10661-10666.

Wong AB, Rutherford MA, Gabrielaitis M, Pangršič T, Göttfert F, Frank T, Michanski S, Hell S, Wolf F, Wichmann C, Moser T (2014) Developmental refinement of hair cell synapses tightens the coupling of $\mathrm{Ca}^{2+}$ influx to exocytosis. EMBO J 33:247-264.

Wu JS, Young ED, Glowatzki E (2016) Maturation of spontaneous firing properties after hearing onset in rat auditory nerve fibers: spontaneous rates, refractoriness, and interfiber correlations. J Neurosci 36:10584-10597.

Young ED, Barta PE (1986) Rate responses of auditory nerve fibers to tones in noise near threshold. J Acoust Soc Am 79:426-442.

Zeddies DG, Siegel JH (2004) A biophysical model of an inner hair cell. J Acoust Soc Am 116:426-441.

Zhang X, Heinz MG, Bruce IC, Carney LH (2001) A phenomenological model for the responses of auditory-nerve fibers: I. Nonlinear tuning with compression and suppression. J Acoust Soc Am 109:648-670.

Zilany MS, Bruce IC, Nelson PC, Carney LH (2009) A phenomenological model of the synapse between the inner hair cell and auditory nerve: long-term adaptation with power-law dynamics. J Acoust Soc Am 126:2390-2412. 